\title{
HP02GO: prediction of human phenotype ontology term associations using cross ontology annotation co-occurrences
}

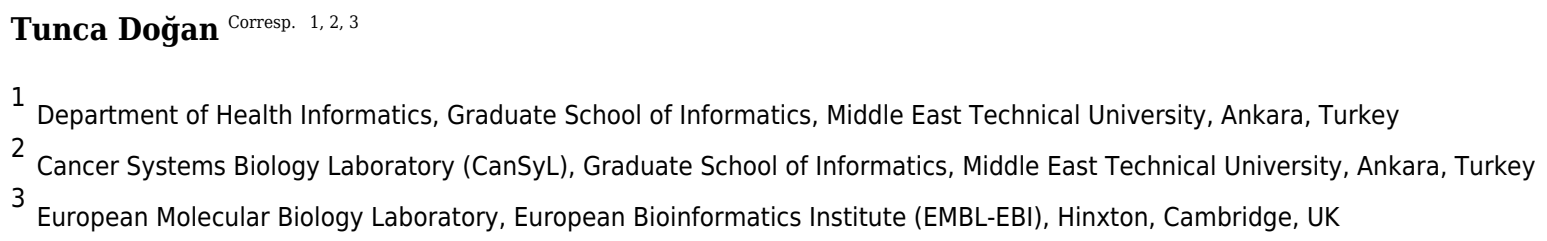

Analysing the relationships between biomolecules and the genetic diseases is a highly active area of research, where the aim is to identify the genes and their products that cause a particular disease due to functional changes originated from mutations. Biological ontologies are frequently employed in these studies, which provides researchers with extensive opportunities for knowledge discovery through computational data analysis. In this study, a novel approach is proposed for the identification of relationships between biomedical entities by automatically mapping phenotypic abnormality defining HPO terms with biomolecular function defining GO terms, where each association indicates the occurrence of the abnormality due to the loss of the biomolecular function expressed by the corresponding GO term. The proposed HPO2GO mappings were extracted by calculating the frequency of the co-annotations of the terms on the same genes/proteins, using already existing curated HPO and GO annotation sets. This was followed by the filtering of the unreliable mappings that could be observed due to chance, by statistical resampling of the co-occurrence similarity distributions. Furthermore, the biological relevance of the finalized mappings were discussed over selected cases, using the literature. The resulting HPO2GO mappings can be employed in different settings to predict and to analyse novel gene/protein - ontology term - disease relations. As an application of the proposed approach, HPO term - protein associations (i.e., HPO2protein) were predicted. In order to test the predictive performance of the method on a quantitative basis, and to compare it with the state-of-the-art, CAFA2 challenge HPO prediction target protein set was employed. The results of the benchmark indicated the potential of the proposed approach, as HPO2GO performed better than all of the models from 38 participating groups (with Fmax $=0.402$ ), by a margin of $12.6 \%$ compared to the top performer. The automated cross ontology mapping approach developed in this work may be extended to other ontologies as well, to identify unexplored relation patterns at 
the systemic level. The datasets, results and the source code of HPO2GO are available for download at: https://github.com/cansyl/HPO2GO. 


\section{HPO2GO: Prediction of Human Phenotype Ontology Term}

2 Associations Using Cross Ontology Annotation Co-occurrences

3

4 Tunca Doğan ${ }^{1,2,3, *}$

5

$6{ }^{1}$ Cancer Systems Biology Laboratory (KanSiL), Graduate School of Informatics, METU, Ankara, $7 \quad 06800$, Turkey

$8{ }^{2}$ Department of Health Informatics, Graduate School of Informatics, METU, Ankara, 06800, 9 Turkey

$10{ }^{3}$ European Molecular Biology Laboratory, European Bioinformatics Institute (EMBL-EBI), 11 Hinxton, Cambridge, CB10 1SD, UK

$12 *$ corresponding author email address: tdogan@metu.edu.tr 


\section{ABSTRACT}

15 Analysing the relationships between biomolecules and the genetic diseases is a highly active area of research, where the aim is to identify the genes and their products that cause a particular disease

17 due to functional changes originated from mutations. Biological ontologies are frequently 18 employed in these studies, which provides researchers with extensive opportunities for knowledge 19 discovery through computational data analysis.

20 In this study, a novel approach is proposed for the identification of relationships between 21 biomedical entities by automatically mapping phenotypic abnormality defining HPO terms with 22 biomolecular function defining GO terms, where each association indicates the occurrence of the 23 abnormality due to the loss of the biomolecular function expressed by the corresponding GO term. 24 The proposed HPO2GO mappings were extracted by calculating the frequency of the co25 annotations of the terms on the same genes/proteins, using already existing curated HPO and GO 26 annotation sets. This was followed by the filtering of the unreliable mappings that could be 27 observed due to chance, by statistical resampling of the co-occurrence similarity distributions. 28 Furthermore, the biological relevance of the finalized mappings were discussed over selected 29 cases, using the literature.

30 The resulting HPO2GO mappings can be employed in different settings to predict and to analyse 31 novel gene/protein - ontology term - disease relations. As an application of the proposed approach, 32 HPO term - protein associations (i.e., HPO2protein) were predicted. In order to test the predictive 33 performance of the method on a quantitative basis, and to compare it with the state-of-the-art, 34 CAFA2 challenge HPO prediction target protein set was employed. The results of the benchmark 35 indicated the potential of the proposed approach, as HPO2GO performance was among the best $36($ Fmax $=0.35)$. The automated cross ontology mapping approach developed in this work may be 37 extended to other ontologies as well, to identify unexplored relation patterns at the systemic level. 38 The datasets, results and the source code of HPO2GO are available for download at: 39 https://github.com/cansyl/HPO2GO. 
40

41

42

43

44

45

46

47

48

49

50

51

52

53

54

55

56

57

58

59

60

61

62

63

64

65

66

67

68

69

70

\section{INTRODUCTION AND BACKGROUND}

Systematic definition of biomedical entities (e.g., diseases, abnormalities, symptoms, traits, gene and protein attributes, activities, functions and etc.) is crucial for computational studies in biomedicine. Ontological systems, composed of standardized controlled vocabularies, are employed for this purpose. Human Phenotype Ontology (HPO) system annotates disease records (i.e., terms and definitions about diseases together with related information) with a standardized phenotypic vocabulary (Robinson et al., 2008; Köhler et al., 2017). HPO is composed of five independent sub-ontologies namely, phenotypic abnormality (i.e., the main sub-ontology defining the basic qualities of diseases), mode of inheritance (i.e., annotates diseases in terms of mendelian or non-mendelian principles), mortality / aging (i.e., information related to age of death due to the corresponding disease), frequency (i.e., frequency of the disease in a patient cohort) and the clinical modifier (i.e., additional disease characterization such as lethality, severity, etc.). Within each subontology, all terms are related to each other with a parent-child relationship, where each child term defines a specific aspect of its parent. HPO has a directed acyclic graph (DAG) structure. The sources of the disease information in HPO are Orphanet (Rath et al., 2012), DECIPHER (Firth et al., 2009), and OMIM (Amberger et al., 2014) databases. Each term in the phenotypic abnormality sub-ontology define a specific type of abnormality encountered in human diseases (e.g., HP:0001631 - atrial septal defect). The generation of HPO terms (and their associations with diseases) are carried out via both manual curation efforts and automated procedures (e.g., text mining). The curation job is usually done by experts by reviewing the relevant literature publications along with the disease centric information at various biomedical data resources. For each association between a disease term and an HPO term, there is an evidence code tag to specify the source of the information (i.e., curated or automated). The evidence codes used in HPO are IEA (inferred from electronical annotation), PCS (published clinical study), ICE (individual clinical experience), ITM (inferred by text mining), TAS (traceable author statement). As of January 2018, the growing library of HPO contains nearly 12,000 phenotype terms, providing more than 123,000 annotations to 7,000 different rare (mostly Mendelian) diseases and the newly added 132,000 annotations to 3,145 common diseases (Groza et al., 2015). A long-term goal of the HPO project is for the system to be adopted for clinical diagnostics. This will both provide a standardized approach to medical diagnostics and present structured machine readable biomedical data for the development of novel computational methods. Apart from phenotype-disease 
71 associations, which is the main aim of the HPO project, HPO also provides phenotype-gene 72 associations by using the known rare disease - gene relations (i.e., the information which is in the 73 form of: "certain mutation(s) in Gene $X$ causes the hereditary Disease $Y ")$, directly using the 74 abovementioned disease centric resources (e.g., Orphanet and OMIM). The disease-gene 75 associations in the source databases are produced by expert curation from the publications of 76 clinical molecular studies. The associations between HPO terms and biomolecules, together with 77 the downstream analysis of these associations, help in disease gene identification and prioritization 78 (Köhler et al., 2009). With the mapping of phenotypes to human genes, HPO currently (January 79 2018) provides 122,166 annotations between 3,698 human genes and 6,729 HPO terms.

80

81

82

83

84 85 86

87 88 89 90 91 92

93

94 95 96 97 98 99 100

The Gene Ontology (GO) is an ontological system to define gene/protein attributes with an extensive controlled vocabulary (GO Consortium, 2014). Each GO term defines a unique aspect of biomolecular attributes. Similar to other ontological systems, GO has a directed acyclic graph (DAG) structure, where terms are related to each other mostly with "is_a" or "part_of" relationships. GO is composed of three categories (i.e., aspects) in terms of the type of the defined gene product / protein attribute such as: (i) molecular function - MF (i.e., the fundamental function of the protein at the molecular level; e.g., GO:0016887 - ATPase activity), (ii) biological process - BP (i.e., the high level process, in which the protein plays a role; e.g., GO:0005975 - carbohydrate metabolic process), and (iii) cellular component - CC (i.e., subcellular location, where the protein carries out its intended activity; e.g., GO:0016020 - membrane). Similar to the other ontological systems, the basic way of annotating a gene or protein with a GO term is the manual curation by reviewing the relevant literature. GO also employs the concept of "evidence codes", where all annotations are labelled with descriptions indicating the quality of the source information used for the annotation (e.g., ECO:0000006 - experimental evidence, ECO:0000501 - IEA: evidence used in automatic assertion). UniProt-GOA (Gene Ontology Annotation) database (Huntley et al., 2015) houses an extensive collection of GO annotations for UniProt protein sequence and annotation knowledgebase records. In the UniProtKB/Swiss-Prot database (i.e., housing manually reviewed protein entries with highly reliable annotation) version 2018_02, there are a total of 2,850,015 GO term annotations for 529,941 protein records; whereas in UniProtKB/TrEMBL database (i.e., housing mostly electronically translated uncharacterized protein entries) version 2018_02, there are a total of $189,560,296 \mathrm{GO}$ term annotations for $67,760,658$ protein records. Most of the 
101 annotations for the UniProtKB/TrEMBL database entries are produced by automated predictions

102 (UniProt Consortium, 2017).

103 Due to the high volume of experimental research that (i) discover new associations between 104 biomolecules and ontological terms, and (ii) produce completely new and uncharacterized 105 gene/protein sequences; curation efforts are having hard time in keeping up with the annotation 106 process. To aid manual curation efforts, automated computational methods come into play. These 107 computational methods exploit the approaches and techniques widely used in the fields of data 108 mining, machine learning and statistics, to produce probabilistic associations between biomedical 109 entities. Critical Assessment of Functional Annotation (CAFA) challenge (Radivojac et al., 2013; 110 Jiang et al., 2016) aims to evaluate the automated methods that produce GO and HPO term 111 association predictions for protein entries, on standard temporal hold-out benchmarking datasets. 112 Now after its third instalment, CAFA organization has already brought together a research 113 community, dedicated to elevate the capabilities of automated function prediction approaches 114 closer to the level of expert review.

115 Protein function prediction using GO terms is a highly active area of research, where various types 116 of approaches utilizing: amino acid sequence similarities (Hawkins et al., 2009), 3D structure 117 analysis (Roy, Yang \& Zhang, 2012), semantic similarities between the ontological terms (Falda 118 et al., 2012), gene expression profiles (Lan et al., 2013), protein-protein interactions - PPIs (Wass, 119 Barton \& Sternberg, 2012), shared functional domains and their arrangements (Fang \& Gough, 120 2012; Finn et al, 2016; Doğan et al., 2016) and ensemble approaches that exploit multiple feature 121 types (Wass, Barton \& Sternberg, 2012; Cozzetto et al., 2013; Lan et al., 2013; Rifaioglu et al., 122 2017); are employed to model the proteins and to transfer the functional annotations from 123 characterized proteins (i.e., the ones that have reliable annotation), to the uncharacterized ones 124 with highly similar features. Known GO associations of genes and proteins are also used in 125 different contexts in the literature. For example, the method "MedSim" uses the semantic 126 similarities between GO terms for the prioritization of disease genes (Schlicker, Lengauer \& 127 Albrecht, 2010). The method "spgk" uses a shortest-path graph kernel to compute functional 128 similarities between gene products using their GO annotations and the term relations on the GO 129 DAG (Alvarez, Qi \& Yan, 2011). 
130 The automated prediction of the associations between human genes/proteins and

131 phenotype/disease defining ontological terms is also a non-trivial task. The resulting predictions

132 can then be utilized to identify large-scale novel disease-gene-pathway/system relations. The

133 identification of direct disease-gene relations is a widely studied topic (Moreau \& Tranchevent,

134 2012). A considerable amount of the existing literature about disease-gene associations involve

135 the calculation of semantic similarities between gene products, based on the already existing

136 ontological term annotations (Washington et al., 2009; Smedley et al., 2013; Deng et al., 2015;

137 Rodríguez-García et al., 2017). For example, the method "PhenomeNET" was employed to

138 generate mappings between the highly related terms across similar ontological systems

139 (Rodríguez-García et al., 2017) such as the HPO, Mammalian Phenotype Ontology - MP (Smith,

140 Goldsmith \& Eppig, 2005), Human Disease Ontology - DO (Kibbe et al., 2014) and Orphanet

141 Rare Disease Ontology - ORDO (Vasant et al., 2014); for discovering novel gene-disease

142 associations. However, semantic similarity based approaches sometimes suffers from the low

143 coverage of the HPO annotations on the protein space. The authors of two recent studies have

144 investigated this issue (Kulmanov \& Hoehndorf, 2017; Peng et al., 2017). In this context,

145 increasing the coverage of HPO annotations by predicting gene/protein-HPO term associations

146 may help semantic similarity based association studies.

147 There are only a few examples of HPO term-protein association prediction methods in the

148 literature. In the "dcGO" method, the authors mapped ontological terms (including HPO) to protein

149 domains, which are the functional units, and transferred the ontology mapping to proteins

150 according to known domain annotations (Fang \& Gough, 2012). The objective in the

151 "PHENOstruct" method is the prediction of gene-HPO term associations using heterogeneous

152 biological data consisting of protein-protein interactions (PPIs), GO annotations, literature

153 relations, variants and known HPO annotations, together with a structured SVM classifier

154 (Kahanda et al., 2015). In this sense, it is interesting see another method that utilize already existing

155 GO annotations to predict HPO terms. Nevertheless, according to the authors, the contribution of

156 the GO features to the overall predictive performance of PHENOstruct was minimal. A text mining

157 based method "EVEX", was employed for protein-HPO term association prediction. Originally,

158 EVEX utilizes text mining approaches for large-scale integration of heterogeneous biological data

159 and event extraction to generate a structured resource of relations, to be used in pathway curation

160 (Van Landeghem et al., 2013). In the context of HPO term prediction, EVEX scans the literature 
161 to detect proteins and phenotypic terms that co-occur on the same text corpus, and associates them

162 with each other based on certain criteria. According to the CAFA2 challenge results (Jiang et al., 163 2016), the participating method EVEX was the top performer. A network based HPO prediction 164 method was the "RANKS", in which the authors developed a flexible algorithmic scheme for 165 heterogeneous biological network analysis, and used previously generated functional Interaction 166 and functional human gene networks for gene-HPO term association prediction (Valentini et al., 167 2016). In a recent study, the authors proposed two hierarchical ensemble methods: (i) the 168 Hierarchical Top-Down, and (ii) the True Path Rule, for gene-HPO term associations; in which 169 the hierarchical graph structure of HPO has been utilized together with the RANKS algorithm and 170 the SVM classifier (Notaro, et al., 2017).

171 The text mining approach is highly effective for predicting gene-disease relations in disease gene 172 prioritization studies (Krallinger, Valencia \& Hirschman, 2008). However, this approach suffers 173 from low coverage in some cases, due to knowledge limitation in the literature. If a certain 174 abnormality and a gene/protein has not been studied together in the same framework yet, it is often 175 not possible to identify the relation. Network based methods are proposed on top of either text176 mining results, protein-protein interactions and/or pathway data (Bromberg, 2013; Guney \& Oliva, 177 2014; Guala \& Sonnhammer, 2017) to detect indirect relations, which greatly increased the 178 coverage; nevertheless, they still moderately rely on the previously reported relations. It is also 179 important to note that, any predictive approach is limited by the quality and the coverage of its 180 source information. However, the predictive output of different approaches often complement each 181 other, contributing to fill different portions of the missing information in the knowledge space. 182 Due to this reason, developing novel approaches to complement the conventional methods is 183 crucial for the automated ontological association prediction. The observed low performance of 184 even the best methods in the HPO term prediction track of the CAFA2 challenge displayed the necessity of novel approaches for the biomedical entity relation prediction.

186 In this study, a new approach "HPO2GO" is proposed to produce phenotypic abnormality HPO 187 term associations to both GO terms and human genes/proteins, with the analysis of co-annotation 188 fractions between the HPO and GO term combinations. For this, HPO and GO terms that are 189 continually co-occurring on different genes/proteins as annotations, are linked to each other (i.e., 190 the system training step), entitled as the "HPO2GO mappings". After that, proteins with a linked 191 GO term annotation receives the corresponding HPO term as the phenotypic term prediction (i.e., 
192 the application step), entitled as the "HPO2protein predictions". The idea here is to associate a 193 HPO term $Y$ with a GO term $X$ in the sense that: "if a protein loses its function defined by the GO 194 term $X$ (or at least a reduction in the defined functionality) as a result of a genetic mutation, the 195 loss of function may cause the disease, which is defined by the phenotype term $Y^{\prime \prime}$. This idea is 196 based on the nature of annotating genes/proteins with HPO terms; for example, only the 197 functionally perturbed variants of these genes/proteins (e.g., disease causing variants) are 198 associated with the relevant genetic diseases and their defining phenotypic abnormality terms. 199 Mutations often lead to diseases by causing either a loss of the existing functionality or a gain of 200 new functionality in the gene products. As a result, if the HPO term $Y$ and the GO term $X$ are 201 observed to be frequently co-occurring on different proteins, then the lost function, which gave 202 way to the corresponding disease may be the one defined by the GO term $X$. This logic would 203 make biological sense especially when the corresponding function is a large-scale biological 204 process. This approach exploits the significantly higher coverage of GO term annotations for 205 genes/proteins, compared to the HPO term annotations; to produce novel gene/protein - HPO term 206 associations.

207 In order to test the biological relevance of this approach, selected HPO2GO mappings were 208 manually examined. Additionally, the proposed methodology was employed to predict HPO terms 209 for the human protein target dataset provided in the CAFA2 challenge. Using the benchmark set, 210 the prediction performance was calculated and compared with the state-of-the-art HPO prediction 211 methods. Another set of HPO2GO mappings were generated for this test, using the temporal hold212 out training data provided in CAFA2. Finally, the up-to-date HPO2GO mappings were employed 213 to generate HPO term predictions to human protein entries in the UniProtKB/Swiss-Prot database 214 (i.e., HPO2protein predictions). The training and test datasets, along with the source code of the 215 proposed methodology and the analyses are available for download at 216 https://github.com/cansyl/HPO2GO. 


\section{2. METHODS}

\section{Dataset Construction}

219 In order to generate the training sets; first, gene to HPO term association file was downloaded from 220 the HPO web-site (January 2017 version of the file named: 221 "ALL_SOURCES_ALL_FREQUENCIES_ genes_to_phenotype.txt"). This file contained 222 153,575 annotations between 3,526 human genes and 6,018 HPO terms. This file is shared in the $223 \mathrm{HPO} 2 \mathrm{GO}$ repository with the filename: 224 "HPO_gene_to_phenotype_annotation_01_2017_ALL_SOURCES_ALL_FREQUENCIES.txt". 225 These gene-phenotype associations are generated as a part of the HPO project by merging two 226 source files: first, the gene-disease associations provided in the incorporated disease centric 227 databases (e.g., OMIM and Orphanet); and second, the disease-phenotype associations generated 228 by HPO. In HPO, "genes_to_phenotype" file only contains the asserted (i.e., specific) annotations 229 to genes; whereas "phenotype_to_genes" file contains all annotations propagated through the root 230 of the HPO DAG, according to the true path rule. As a result, parents of the asserted terms are 231 included as well. In this study, the asserted annotations are used (in terms of HPO) at the input 232 level, in order to avoid the propagation of potential false positive annotations that may be presented 233 in the source dataset. This application usually comes with the cost of increased number of false 234 negatives; however, avoiding the potential false positives is considered more important here.

235 In order to generate the second training dataset, all GO term annotations to the human proteins in 236 UniProtKB with the manually assigned (curator assigned) evidence codes (i.e., EXP, IDA, IPI, 237 IMP, IGI, IEP, IBA, IC, IKR, ISS, NAS, ND and TAS) were downloaded from the UniProt-GOA 238 database 201701 version, using the QuickGO browser (filename: 239 "GOA_UniProt_human_protein_annotation.tsv"). The reason behind not using electronically 240 assigned annotations (i.e., evidence code: IEA) was that, these annotations have a reduced level of 241 annotation reliability compared to the curator assigned ones; as a result, they may contain 242 erroneous cases (i.e., false positives). After eliminating the repeating (i.e., redundant) annotations, 243 the finalized file contained 179,651 GO annotations between 18,577 unique human genes and 244 14,632 GO terms (filename of the finalized GO annotation file: 245 "GO_annot_human_proteins_UniProtGOA_01_2017.txt"). An additional column containing the 246 corresponding HGNC symbols (i.e., gene symbols) of the coding genes was also included in the 
247 downloaded GO annotation file. This column was later used to combine the GO annotations with

248 the HPO annotations, since the HPO annotation file includes the gene symbols.

249

\section{Applied Methodology}

251 The proposed methodology is divided into 2 steps: (i) training of the system (i.e., the generation 252 of the HPO2GO mappings), and (ii) the application step (i.e., the prediction of HPO term-protein 253 associations - HPO2protein, using the previously generated HPO2GO mappings).

254 Figure 1 represents the complete HPO2GO mapping (i.e., training) procedure. For the training of 255 the system, first, the HPO and GO annotation datasets were prepared (Figure 1.A and Figure 1.B) 256 and the initial HPO-GO mappings were generated (Figure 1.C) by identifying the genes/proteins 257 shared between individual HPO and GO terms (i.e., the cases where HPO and GO terms were co258 annotated to the same genes/proteins). This mapping has generated 1,433,208 unique pairs 259 between 6,005 HPO terms and 9,685 GO terms. At this point, it was observed that some of GO 260 and HPO terms were annotated to high number of proteins, and it was highly probable for them to 261 co-occur on the same protein once or twice just by chance. In order to eliminate these cases, a 262 filtering procedure was required. For each HPO-GO term pair, a co-occurrence similarity measure, 263 inspired from an information-theoretic definition of similarity (Lin, 1998), has been calculated. 264 The co-occurrence similarity formulation is given in Equation 1.

265

266

$$
S_{H P O i, G O j}=\frac{2 * N_{G H P O i \& G O j}}{N_{G H P O i}+N_{G G O j}}
$$

267

268 Here, $S_{H P O i, G O j}$ is the co-occurrence similarity between the HPO term "HPOi" and the GO term 269 "GOj", $N_{G H P O i \& G O i}$ is the number of genes/proteins where these terms are annotated together, $N_{G}$ 270 $\mathrm{HPOi}$ is the total number of genes with the annotation "HPOi", and $N_{G G O i}$ is the total number of 271 genes with the annotation "GOi".

272 The mapping process and the co-occurrence similarity calculation are shown in Figure 2 with a 273 toy example. Following the calculation of the co-occurrence similarities between all HPO-GO 274 pairs, a thresholding operation was applied in order to distinguish between relevant mappings and 275 the random ones. Two parameters were used for the thresholding operation: $(i)$ the co-occurrence 
276 similarities: $S$, and (ii) the number of genes with co-occurring annotations: $n$. The aim behind 277 employing a second parameter (i.e., $n$ ) was to eliminate the potential random pairing cases, where 278 the co-occurrence similarity is still high. These cases are rare; however, it is still possible to 279 observe a few of them especially when $n$ is very small, due to extremely high number of term 280 combinations. In Figure 2, this situation is represented on the toy example, here $S_{H P O D, G O 4}$ is equal 281 to $S_{H P O B, G O 3}$; however the $\mathrm{HPO}_{D^{-}} \mathrm{GO}_{4}$ mapping is probably less reliable compared to $\mathrm{HPO}_{B^{-}} \mathrm{GO}_{3}$ 282 since $n_{H P O D, G O 4}$ is equal to 1.

283 Statistical resampling was used to determine the optimal parameter values (to be used as 284 thresholds), that separate meaningful mappings from random ones. A permutation (i.e., 285 randomization) test was constructed for this purpose. At this point, a randomized HPO-GO term 286 mapping table was required, which was generated (Figure 1.D) by first, shuffling the indices of 287 the original "HPO vs. gene" and "GO vs. gene" annotation tables; and second, calculating both the 288 randomized co-occurrence similarities (i.e., $S_{R}$ ) and the number of genes with co-occurring 289 annotations (i.e., $n_{R}$ ) for each random HPO-GO mapping. For each arbitrarily selected $S$ (i.e., $S>$ $2900, S \geq 0.1, S \geq 0.2, \ldots, S \geq 0.6$ ) and $n$ (i.e., $n \geq 1, n \geq 2, \ldots, n \geq 5$ ) threshold value combination, the 291 original GO-HPO mappings with lower than the threshold $S$ and $n$ values were deleted and a co292 occurrence similarity distribution histogram was plotted using the remaining mappings (i.e., 293 histograms plots in Figure 1 and in Figure 3). The same procedure was applied for the randomized 294 mapping set as well. Finally, Kolmogorov-Smirnov test -KS test- (Lilliefors, 1967; Hollander, 295 Wolfe \& Chicken, 2013) is employed to calculate a test statistic for estimating whether the samples 296 from the random and the original sets (at each $S$ and $n$ selection) are from the same distribution or 297 not. KS is a nonparametric test of 1-dimensional probability distributions that can be used to 298 compare two samples, considering the quantized distance between the samples. The null 299 hypothesis states that the two samples are drawn from the same distribution. Here, the distribution 300 (i.e., histogram) of the $S$ values for the original and the randomized mapping sets represent the two 301 samples. The reason behind using histograms instead of the actual $S$ values was that, both high and 302 low $S$ values were presented in both distributions; as a result, the significance test by checking 303 sample distances approach would not work. However, the frequencies of these high and low $S$ 304 values are different from each other in the original and the random distributions. If the null 305 hypothesis is accepted at a selected threshold value pair ( $S$ and $n$ ), which means that the 306 distributions are not statistically different from each other, then it is concluded that the selected 
307 thresholds failed to eliminate the random pairings in the original mapping (i.e., a higher threshold

308 is required). The lowest threshold values, where the samples from the two distributions became

309 significantly different from each other, were selected as the official thresholds. Excessive threshold

310 values were not considered in order not to eliminate too many GO-HPO mappings. After the

311 determination of the parameter values (i.e., $S$ and $n$ thresholds), the HPO2GO mappings were

312 finalized, which ended the training process.

313 An alternative to the statistical resampling methodology used here would be employing a direct

314 approach such as an exact test (e.g., Fisher's exact test), and to calculate a significance value for 315 each HPO-GO mapping individually. The advantage of this approach would be accepting or 316 rejecting the null hypotheses specific to each mapping (as opposed to finding a global significance

317 using the whole data at once, as applied in this study). The main disadvantage of this approach 318 would be the diminished statistical power to calculate significance values for the mappings where 319 the number of instances are too low. Unfortunately, this is the case for most of the HPO-GO 320 mappings as the mean number of occurrences (for all HPO-GO mappings) in the original or in the 321 random sets were 1.77 and 1.48, respectively. As a result, the direct approach was not considered 322 in this study.

323 HPO2protein prediction step was a simple procedure, where query proteins were annotated with 324 the HPO terms, by taking their already existing GO annotations into account. HPO2GO mappings 325 were employed for this purpose. There were a total of 3 application runs in this study using: (i) 326 CAFA2 targets as the query set (for the performance tests and for the comparison with the state327 of-the-art), (ii) CAFA3 targets as the query set (to officially participate in the CAFA3 challenge), 328 and (iii) all human protein entries in the UniProtKB/Swiss-Prot database (to generate the $329 \mathrm{HPO} 2$ protein predictions).

\section{Performance Evaluation Metrics}

332 In this study, it was not possible to use a standard fold based cross-validation to measure the 333 performance and to determine the parameter values in the training procedure, since in most cases, 334 the number of genes/proteins that have a co-occurring HPO-GO term annotations were extremely 335 low. As a result, it was not impossible to separate the samples into training and validation sets. 336 Instead, the optimal parameter values were determined by using statistical resampling. However, 
337 a performance test was still required in order to assess the success of the proposed approach. For 338 this, CAFA2 challenge benchmark set was employed. Due to the fact that CAFA2 challenge was 339 long before the analysis done in this study, HPO2GO mappings were re-generated using both the $340 \mathrm{GO}$ and HPO annotation data from January 2014. This was followed by the production of the 341 HPO-protein association predictions on the CAFA2 target gene set. This analysis both served as a 342 performance test with the temporal hold-out data (one of the hardest and most informative tests 343 for predictive models) and a performance comparison with the state-of-the-art (i.e., other HPO 344 prediction methods participated in CAFA2). The most basic definitions of the evaluation metrics 345 used in this test; recall, precision, Fmax and minimum semantic distance (Smin) are shown in 346 Equation 2, 3, 4 and 5.

347

348

349

350

351

352

353

354

355

356

357

358

359

360

361

362

363

364

365

$$
R c_{\tau i}=\frac{T P_{\tau i}}{T P_{\tau i}+F N_{\tau i}}
$$

$$
F_{\text {max }}=\max _{i=1 \ldots N}\left\{\frac{2 * P r_{\tau i} * R c_{\tau i}}{P r_{\tau i}+R c_{\tau i}}\right\}
$$

$$
S_{\min }=\min _{i=1 \ldots N}\left\{\sqrt{R u_{\tau i}^{2}+M i_{\tau i}^{2}}\right\}
$$

In equations 2,3 and 4; $T P_{\tau i}, F N_{\tau i}, F P_{\tau i}, R c_{\tau i}$ and $P r_{\tau i}$ represent the number of true positives, the number of false negatives, the number of false positives, recall and precision values, respectively; at the $i^{\text {th }}$ probabilistic score threshold $\left(\tau_{i}\right)$. Fmax correspond to the maximum of the $F$-score values (i.e., harmonic mean of precision and recall, shown inside the curly brackets in Equation 4) calculated for each arbitrarily selected probabilistic score threshold. $i=1 \ldots N$ represents there are $N$ different arbitrarily selected probabilistic score thresholds. Higher Fmax values indicate higher performance. $R u_{\tau i}$ and $M i_{\tau i}$ in Equation 5 corresponds to remaining uncertainty and normalized misinformation at the $i^{\text {th }}$ probabilistic score threshold $\left(\tau_{i}\right)$, respectively. Smin is the minimum semantic distance. Lower Smin indicate higher performance. Also, a weighted version of the Fmax measure has been calculated. Weighting procedure was applied using the information content of 
366 each ontology term; so that, more informative terms obtained higher weights. Information

367 regarding the calculation of remaining uncertainty, misinformation and term based information

368 contents can be found in Clark and Radivojac, 2013 and in Jiang et al., 2016.

369 In the proposed method, probabilistic scores for each HPO-protein association prediction is

370 calculated using the term co-occurrence similarity scores in Equation 1. If the mapping between

371 the terms $H P O i$ and $G O j$ received the co-occurrence similarity score $S_{H P O i, G O j}$, then all proteins

372 that receive the $H P O i$ prediction due to the presence of GOj annotation obtain the probabilistic

373 prediction score: $S_{H P O i, G O j}$. The calculation of the score in Equation 1 is set to range between 0

374 and 1; as a result, it can directly be used as a probabilistic score. Apart from that, probabilistic

375 score thresholds represent values, under which the predictions are discarded. This way, a different

376 set of predictions are given for each arbitrarily selected probabilistic score thresholds, leading to

377 different precision and recall values. It is important to note that, probabilistic score thresholds are

378 different from the thresholds we used to filter out unreliable HPO2GO mappings during the

379 training process. The probabilistic score thresholds are used here (i.e., after the production of

380 HPO2protein predictions) to produce binary predictions from continuous prediction scores, to be

381 able to calculate performances. More details regarding the CAFA2 evaluation metrics are given in

382 Jiang et al., 2016. 


\section{3. RESULTS}

\section{Statistical Analysis of the Mappings}

386 The initial HPO to GO mappings were generated according to the procedure explained in the 387 Methods section (Figure 2). The initial mapping of the original set resulted in 1,433,208 mappings 388 between 6,005 HPO terms and 9,685 GO terms. The same procedure for the randomized set 389 produced 1,543,917 mappings between 5,995 HPO terms and 9,685 GO terms. The initial HPO-

390 GO mappings for both the original and the randomized sets are available for download in the 391 repository of the study (respective filenames: "HPO_GO_Raw_Original_Mapping.txt" and 392 "HPO_GO_Random_Mapping.txt"). It was expected that the mappings generated from the random 393 set would have lower co-occurrence similarity values on average compared to the original set

394

395

396

397

398

399

400

401

402

403

404

405

406

407

408

409

410

411

412 mappings; in other words, they would contain less number of mappings for a particular cooccurrence similarity value. Table 1 displays the comparison of the number of mappings for different co-occurrence similarity values, between the original and the randomized sets. As observed from Table 1, when $S>0$ there is no difference between the mappings; however as $S$ is increased, the difference between the mappings becomes clear. Also, when $S$ is increased, the number of mapped HPO and GO terms were decreased since many terms did not have any mappings that satisfied the stringent $S$ values. The parameter $n$ was not taken into account while calculating the statistics in Table 1 (i.e., $n \geq 1$ for all values in the table).

The histograms in Figure 3 display the co-occurrence similarity distributions (i.e., $S$ ) for arbitrarily selected $n$ values. As observed from the histograms, when the mappings with low $n$ values are eliminated, the distributions shift to the right (i.e., the mean of $S$ increases), which can be interpreted as the mappings became more reliable. However, excessive values of $n$ thresholds leave only a few mappings to work with, especially at $n=25$ and $n=75$ (please see the number of mappings at the vertical axis of Figure 3.C and D). Histograms in Figure 3 also show that thresholding the mappings using only $n$ (not using $S$ at all) would not be sufficient because there are mappings with very low $S$ values even at very high $n$ thresholds (i.e., 25 and 75). This observation verified the decision to use both of the parameters for the filtering operation. At this point, the statistical resampling (i.e., KS test) was applied since it was not possible to determine the optimal $n$ threshold by just manually checking the histograms. 
413 In order to find the minimum $S$ and $n$ values that significantly separate the original mapping from 414 the randomized mapping, 35 different distributions, all combinations of the selected $n$ (i.e., $n \geq 1$, $4152, \ldots, 5)$ and $S$ (i.e., $S>0, S \geq 0.1, \ldots, 0.6$ ) values, were prepared and tested individually against 416 the co-occurrence distribution of the random mapping, generated with the same $S$ and $n$ thresholds. 417 This test resulted in 35 different $p$-value calculations and the minimum parameter values that 418 satisfied the statistical significance (i.e., rejection of the null hypothesis, which states that the two 419 samples are from the same distribution) were selected. Table 2 displays the significance results of 420 all KS tests. The cells with "NaN" indicate the cases, where the test could not be completed due 421 insufficient number of samples to calculate the statistic. However, incomplete tests were not a 422 problem since the aim here was observing the minimum threshold values, where the distributions 423 significantly diverge from each other (NaNs are located far away from this point). In Table 2, the 424 cell with the $p$-value written in bold font (i.e., 0.0057) signifies the point, where the corresponding 425 thresholds $n \geq 2$ and $S \geq 0.1$ yielded the required significance ( $p$-value $<0.01$ ); and thus, these 426 values were selected as the finalized thresholds. This means that, all of the mappings with $n<2$ 427 and $S<0.1$ were considered unreliable and eliminated from the initial HPO-GO mappings.

428 Figure 4 displays the total number of unique mappings (vertical axis) with co-occurrence similarity 429 values greater than the corresponding threshold value (horizontal axis), for the original and the 430 randomized distributions on the blue and red coloured curves, respectively. Figure 4.A shows the 431 plot for the combination with greater than or equal to one co-annotated gene (i.e., $n \geq 1$ ), Figure 432 4.B displays the same value for $n \geq 2$, Figure 4.C and $\mathrm{D}$ for $n \geq 3$ and 4; respectively. The 433 differences between Figure 3 and Figure 4 is that, (i) in Figure 4 cumulative number of mappings 434 are given (i.e., all mappings left after thresholding with $S \geq 0.1,0.2, \ldots$ ), whereas in Figure 3 , the 435 number of mappings that fall into each $S$ bin is given; and (ii) in Figure 4, plots are given for $n \geq$ $4361,2,3$ and 4 since the aim was to display the curves around the selected threshold $n$ value; whereas 437 in Figure 3, there are plots for $n \geq 1,5,25$ and 75 to visually indicate the distribution shifts 438 especially at high $n$ values (i.e., $n=25$ and $n=75$ ). Figure 4 was drawn as a visual representation of 439 the likeness between the original and the randomized distributions at different parameter 440 selections. As observed from Figure 4, the distributions diverged from each other at $n \geq 2$, which 441 also is consistent with the KS test results. Considering the co-occurrence similarity parameter, $S \geq$ 4420.1 produced a clear separation between the original and the randomized distributions as long as $n$ 443 is greater than 1. Following the HPO-GO mapping elimination according to the selected 
444 thresholds, finalized HPO2GO mappings contained 45,805 associations between 3,693 HPO terms 445 and 2,801 GO terms. HPO2GO mappings are available for download in the repository of the study 446 (filename: "HPO2GO_Finalized_Mapping.txt").

447 It was only possible to use a small portion of the input GO annotations for the generation of the 448 HPO2GO mappings because the number of HPO annotated genes were only 3,526; whereas, the 449 number of GO annotated human genes were 18,577. Since mappings can be done over the 450 genes/proteins with co-occurring GO and HPO annotations, only 3,526 genes/proteins were used 451 in the process. The remaining 15,051 human genes with GO annotations were only used in the 452 application step (i.e., HPO2protein), to predict HPO term associations.

453

454 Comparison of HPO2GO with the manual HPO-GO associations

455 As a part of the main HPO project, a sub-set of the HPO terms has been mapped to the relevant 456 terms from different ontologies (e.g., anatomy, Gene Ontology process or cell type) to yield 457 semantic interoperability with these systems. Finalized HPO2GO mappings were compared with 458 these manual GO associations in order to observe the correspondence. The manual mappings are 459 available in the "hp.owl" ontology file in the HPO repository. From the most up to date version 460 (03_2018) of the owl file, the corresponding associations have been extracted and stored in the 461 HPO2GO repository with the filename: "HPO_manual_GO_associations_03_2018.txt". There 462 were 489 manual associations between 488 HPO terms and 239 GO terms, which was significantly 463 lower compared to the HPO2GO mappings (i.e., 45,805 mappings between 3,693 HPO terms and 464 2,801 GO terms). Considering the most frequent GO terms in these manual associations, 71 HPO 465 terms were associated with the GO term "ossification" (GO:0001503), 51 HPO terms were 466 associated with the GO term "inflammatory response" (GO:0006954) and 37 HPO terms were 467 associated with the GO term "pigmentation" (GO:0043473). The comparison of manual 468 associations with HPO2GO mappings have revealed that nearly $40 \%$ of the HPO terms in the 469 manual associations were also mapped to at least one GO term in HPO2GO. When the same 470 calculation was done to reveal how many of the HPO terms in HPO2GO were also in the manual 471 associations, the result was only 5\%. The same correspondence results for GO terms were 35\% 472 and 3\%, respectively. Finally, the correspondence between the actual HPO-GO mappings has been 473 calculated. Only 23 out of 489 manual associations were retrieved by HPO2GO. An inspection 
474 was done to reveal the possible reasons behind the low correspondence, and it was found that, the

475 low number of gene annotations of either the corresponding HPO term or the GO term was the

476 main reason. However, in many cases, an ancestor of the corresponding HPO or GO term was able

477 to be mapped. For example, there was a manual association between "epididymitis: the presence 478 of inflammation of the epididymis" (HP:0000031) and "inflammatory response" (GO:0006954). 479 HPO2GO managed to retrieve an association between "epididymitis" (HP:0000031) and "defense 480 response" (GO:0006952), which is the direct parent term (with "is_a" relationship) of 481 "inflammatory response" (GO:0006954). The "inflammatory response" GO term could not be 482 mapped to any HPO term by HPO2GO since this term was not directly annotated to any 483 gene/protein in UniProt-GOA.

484

485

\section{Performance Comparison with the State-of-the-art}

486 The test for the comparison with the state-of-the-art had two objectives: (i) measuring the 487 performance of the method on a temporal hold-out dataset to observe the relevance of the proposed 488 approach, and (ii) investigating how the proposed method competes with the best performing 489 methods in the literature. For this, we have re-generated the HPO2GO mappings using the CAFA2 490 training set, which contained 133,175 annotations between 5,586 HPO terms and 4,418 proteins, 491 from January 2014. Whereas, CAFA2 evaluation set (i.e., the benchmarking set) contained 19,743 492 annotations between 1,845 HPO terms and 238 proteins (considering only the no-knowledge 493 benchmark samples). The reason behind the presence of low number of annotations (and proteins) 494 in the evaluation set was that, only the HPO annotations produced between the time of the 495 challenge participation deadline and the end of the annotation collection period (a total duration of 496 nearly 8 months) were used to generate the temporal hold-out evaluation set. One important 497 observation about the benchmark set is that, a few HPO terms dominates the benchmark set (i.e., 498 the most frequently annotated 185 terms own 55\% of all annotations). Also, most of these HPO 499 terms were generic (e.g., HP:0000707 - Abnormality of the nervous system). The small size of the 500 benchmark dataset, together with the uneven distribution of the term frequencies limits the 501 evaluative capacity of this set. All of the datasets, the source code and the supplementary files used 502 in the CAFA2 challenge, and thus in this benchmarking experiment, is available through the CAFA 503 project repositories (URLs: https://github.com/yuxjiang/CAFA2 and 
504 https://ndownloader.figshare.com/files/3658395). The CAFA performance evaluation scripts 505 published in these addresses were directly used to calculate the predictive performance of $506 \mathrm{HPO} 2 \mathrm{GO}$.

507 HPO2GO mappings generated using the CAFA2 training set contained 27,424 mappings between 508 2,640 HPO terms and 2,488 GO terms. Considering the whole CAFA2 human target protein set, 509 this mapping produced 1,922,333 HPO predictions for 16,256 proteins and 2,640 HPO terms. The 510 calculated performance of this prediction set was low $(F \max =0.30)$, mainly due to high number 511 of false positive (FP) hits. However, it is probable that many of these false positives were actually 512 non-documented HPO annotations of the corresponding protein, as the benchmark annotation set 513 is incomplete. Increasing the thresholds with the aim of reducing the number of false positives 514 resulted in a matching increase in the number of false negatives (FN), with a similar Fmax value. 515 With the aim of enriching the mappings (to be able to reduce FPs without a significant increase in 516 FNs), HPO annotations of genes from January 2014 (i.e., the CAFA2 training set) were propagated 517 to the root of HPO DAG according to the true path rule. The propagated training set contained 518379,513 annotations between 4,418 human proteins and 6,576 HPO terms; as opposed to 133,175 519 annotations between 4,418 human proteins and 5,586 HPO terms in the asserted CAFA2 set. As 520 observed from the dataset statistics, propagating the annotations have only added about one 521 thousand new terms to the set; however, the number of annotations were significantly increased. 522 Repeating the CAFA2 benchmark analysis using propagated HPO annotations and the same GO 523 annotations set resulted in the same performance $(F \max =0.30)$. Next, automated GO annotations 524 (i.e., evidence code: IEA) have been included in the source GO annotation set, which increased 525 the number of unique GO annotations from 128,947 to 214,235 (a $66 \%$ increase). Using the 526 propagated HPO annotations together with enlarged GO annotation set, the new HPO-GO 527 mappings, namely "HPOprop2GOall", were generated. The finalized HPOprop2GOall contained 528 198,928 mappings between 4,780 HPO terms and 5,196 GO terms; as opposed to 27,424 mappings 529 between 2,640 HPO terms and 2,488 GO terms in the original mappings. The drastic difference 530 between the numbers have indicated the enrichment provided by annotation propagation and GO 531 set enlargement. Subsequently, HPOprop2GOall mappings were used to predict HPO associations 532 for all CAFA2 targets, producing 13,022,574 predictions (as opposed to 1,922,333 predictions 533 with the asserted set). Considering only the CAFA2 benchmark proteins, the predictions generated 534 by using the optimized parameters (i.e., $n=170$ and $S=0.11$ ) resulted in 34,486 HPO predictions 
535 for 221 benchmark proteins and 235 HPO terms, with a performance of Fmax $=0.35$ (no536 knowledge benchmark sequences in the full evaluation mode), which is among the top 537 performances considering all of the models from 38 participating groups in the CAFA2 HPO 538 prediction track. The Fmax performance of the top model in the challenge was 0.36 (Jiang et al., 539 2016), and the performance of the naïve baseline classifier was also the same. In Figure 5, each 540 bar displays the overall performance (Fmax) of the CAFA2 participators, baseline classifiers and 541 HPO2GO. Additionally, weighted precision-recall curves were plotted, to assess the performance 542 of the method at different threshold selections (Figure 6). The weighting procedure was done 543 according to the information content of each HPO term; as a result, informative terms received 544 higher weights. The term weights were officially calculated and published by CAFA2 challenge 545 evaluators and these weights were directly used in this study. As shown by the black curve in 546 Figure 6, HPO2GO performed the same as the best methods on the optimal point ( $w F \max =0.29)$, 547 indicated by the circular marking over the curve. Furthermore, the minimum semantic distance 548 (Smin) was calculated for HPO2GO and compared again with the CAFA2 participators (Figure 7). 549 Here, lower Smin values indicate higher performance. As shown in Figure 7, HPO2GO performed 550 slightly worse $(\operatorname{Smin}=57.2)$ compared to the top performing methods and the naïve classifier. 551 Finally, term-centric predictive performance of HPO2GO was measured. In the term-centric 552 evaluation, the performance of a predictor is measured independently for each ontology term. 553 Figure 8 displays the average HPO term-centric area under the ROC curve (AUROC) measures

554 for HPO2GO and the CAFA2 participating methods. In this evaluation mode, HPO2GO came 555 second (AUROC $=0.59$ ), with a significantly better result compared to the baseline classifiers. 556 HPO2GO CAFA2 benchmark predictions are available in the repository of the study (filename: 557 "HPO_CAFA2_benchmark_predictions.txt").

558 The coverage of HPO2GO on the CAFA2 benchmark protein set was nearly 97\%. The high 559 coverage indicates that HPO2GO managed to annotate a wide range of proteins. According to the 560 analysis of 6 genes, which codes for the proteins in the CAFA2 benchmark set that HPO2GO could 561 not annotate (gene symbols: ATOH7, DMP4, GNT2C, CE126, PGAP3, SERAC1), only two of 562 these proteins have GO annotations (gene symbols: ATOH7, PGAP3), and the others had no GO 563 annotations at all with experimental evidence codes; as a result, it was impossible for HPO2GO to 564 assign HPO terms for those proteins. Considering ATOH7 and PGAP3, their annotated GO terms 565 (GO:0003407 - neural retina development and GO:0021554 - optic nerve development for ATOH7, 
566 GO:0016788 - hydrolase activity, acting on ester bonds, GO:0006505 - GPI anchor metabolic

567 process and GO:0031227 - intrinsic component of endoplasmic reticulum membrane for PGAP3)

568 have been associated with HPO terms in the raw HPO-GO term mappings; however, all of these

569 mappings were eliminated at the resampling step due to low $n$ and $S$ values.

570 At this point in the study, CAFA2 benchmark performance test was also repeated using the raw

571 HPO-GO mappings (without statistical resampling) in order to observe the impact of eliminating

572 unreliable term mappings using $n$ and $S$ thresholds. The raw mappings were composed of 879,873

573 HPO-GO term associations, which led to 66,522,438 predictions between 18,155 proteins and

5745,559 HPO terms. The statistics indicate that nearly $65 \%$ all possible combinations between the

575 target proteins and HPO terms were produced as predictions. The performance analysis of this

576 prediction set resulted in a very low Fmax value (i.e., 0.001), as expected, indicating the

577 effectiveness of the statistical resampling procedure applied in this study. At all stages of the

578 performance analysis, different HPO2GO mapping sets were generated using various resampling

579 parameters (i.e., different $n$ and $S$ threshold selections), and tested on the CAFA2 benchmark;

580 however, these mappings produced performances slightly inferior to the ones reported above. The

581 most probable reason behind observing reduced performance with a lowered threshold was the

582 inclusion of high number of mappings in the finalized set, most of which were false positives. This

583 in turn provided a reduced precision and a reduced Fmax measure. On the other hand, when

584 excessively high thresholds were selected, many of the reliable mappings were probably discarded,

585 leading to high number of false negatives, thus a reduced recall and a reduced Fmax, as well.

586

587 Generation of the Finalized HPO2protein Predictions

588 Up-to-date HPO2GO mappings were employed to predict HPO terms for the human protein entries 589 in the UniProtKB/Swiss-Prot database (i.e., 20,258 protein records), and the resulting prediction 590 set was marked as the finalized HPO2protein predictions. This set contained 3,468,582 HPO 591 predictions for 18,101 proteins and 3,693 HPO terms. HPO2protein predictions are available in 592 the repository of the study (filename: "HPO2protein_Predictions.txt").

593 Finally, up-to-date HPO2GO model was run on the CAFA3 human protein targets, which produced $5943,453,130$ predictions on 16,609 human proteins with 3,719 HPO terms. A more stringent subset 595 of this prediction set (i.e., predictions produced from mappings with $S \geq 0.2$ ) has been officially 
596 submitted to the CAFA3 challenge. HPO2GO CAFA3 target predictions are available in the 597 repository of the study (filename: "HPO_CAFA3_target_predictions.txt"). There was a small 598 difference between the number of query proteins in HPO2protein and the CAFA3 target sets 599 (20,258 as opposed to 20,197, respectively). At the time of writing this manuscript, the CAFA3 600 challenge results have not been announced yet.

601

602 The Biological Relevance of the Selected HPO2GO Mappings - A Case Study

603 In order to discuss the biological relevance of HPO2GO mappings, selected HPO-GO term 604 mappings were examined. For this purpose, 3 confidence bins (high-level, mid-level and low-level 605 reliabilities) were determined, considering their respective $S$ and $n$ values (high $S$ and $n$ values 606 together indicate elevated reliability). Six example mappings (2 from each confidence bin) were 607 randomly selected for the case study. The first case was a highly reliable mapping between the 608 phenotypic abnormality HPO term "absence of bactericidal oxidative respiratory burst in 609 phagocytes" (HP:0002723) and the GO term "respiratory burst" (GO:0045730), which is in the BP 610 category. The exact definition of this GO term in the UniProt-GOA database is: "A phase of 611 elevated metabolic activity, during which oxygen consumption increases; this leads to the 612 production, by an NADH dependent system, of hydrogen peroxide (H2O2), superoxide anions and 613 hydroxyl radicals." (URL: https://www.ebi.ac.uk/QuickGO/term/GO:0045730). These two terms 614 were mapped to each other in HPO2GO with high confidence (i.e., $S=0.89$ and $n=4$ ). The 615 symbols of the co-annotated genes were $C Y B A, C Y B B, N C F 2$ and $N C F 1$. As observed from the 616 names of both terms and from the description of the GO term, the HPO term defines an abnormal 617 condition that corresponds to the absence of the biological process portrayed by the mapped GO 618 term. This is in accordance with the logic behind mapping HPO terms with GO terms, which stated 619 the occurrence of an abnormality (i.e., the HPO term) due to the loss of the biomolecular function 620 defined by the mapped GO term. In addition, there is a GO term named "respiratory burst after 621 phagocytosis" (GO:0045728), which is related (i.e., is_a relationship) to GO:0045730 as its child 622 (descendant) term. These 2 terms are two-step away from each other on the GO DAG. This term 623 (GO:0045728) defines a more specific function that is the exact opposite of the mapped HPO term 624 (HP:0002723), semantically. Also, there is an evidence for the relation between HP:0002723 and 625 GO:0045728 in both the OBO and OWL formatted ontology files of HPO (URL: 
626 http://purl.obolibrary.org/obo/hp.obo and http://purl.obolibrary.org/obo/hp.owl). However, in 627 HPO2GO, GO:0045728 could not be mapped to HP:0002723 due to low coverage in the source 628 GO annotation set. GO:0045728 was only annotated to one gene (symbol: HCK), which was not 629 annotated to HP:0002723, as a result, the mapping could not be generated. Nevertheless, the 630 mapped GO term (GO:0045730) still defined a sufficiently related function.

631 The second selected case with high-level reliability was the mapping between the HPO term 632 "cerebellar hemisphere hypoplasia" (HP:0100307) and the MF category GO term "tRNA-intron 633 endonuclease activity" (GO:0000213). The exact definition of this specific GO term in the 634 UniProt-GOA database is: "Catalysis of the endonucleolytic cleavage of pre-tRNA, producing 5'635 hydroxyl and 2',3'-cyclic phosphate termini, and specifically removing the intron" (URL: 636 https://www.ebi.ac.uk/QuickGO/term/GO:0000213). These two terms were mapped to each other 637 in HPO2GO with high confidence (i.e., $S=0.86$ and $n=3$ ). The symbols of the co-annotated genes 638 were TSEN2, TSEN34 and TSEN54. The HPO term HP:0100307 is associated with the disease 639 entry "Pontocerebellar Hypoplasia, Type 2C (PCH2C)" (OMIM:612390) in the OMIM database. 640 According to the disease definition, pontocerebellar hypoplasia is a heterogeneous group of 641 neurodegenerative disorders associated with abnormally small cerebellum and brainstem, and the 642 type 2C is characterized by a progressive microcephaly from child birth (Barth, 1993). The 643 occurrence of the disease is associated with missense mutations in either TSEN2, TSEN34 or 644 TSEN54 genes, which are parts of the tRNA splicing endonuclease complex (Budde et al., 2008). 645 It was reported that, due to the abovementioned mutations, there was a partial loss in the function 646 of cleaving the pre-tRNAs by the endonuclease complex (Budde et al., 2008). This is another clear 647 example for a HPO term defining an abnormal condition, that is caused by the perturbation in the 648 function defined by the mapped GO term.

649 An example with mid-level reliability (with parameter values: $0.7>S>0.5$ and $10>n>2$ ), where 650 HPO2GO produced a mapping with reduced biologically relevance is the case where "protein 651 binding" (GO:0005515) MF GO term was associated with "cognitive impairment" (HP:0100543) 652 phenotype term. The parameter values for this mapping were $S=0.38$ and $n=571$. It might be 653 possible to find an indirect connection between some of the diseases that cause cognitive 654 impairment and the loss of protein binding function; however, it is impossible to conclude that all 655 protein binding function losses would result in cognitive impairment. Both of these terms are quite 
656 generic; as a result, the mapping is not informative. The interesting observation here is the 657 extremely high $n$ value, which is resulted from the fact that protein binding GO term is a generic 658 and frequent term that is annotated to many different protein entries. This result also indicated that 659 using the $n$ value alone to judge the reliability of a mapping would not be sufficient, instead both $660 S$ and $n$ should be taken into account.

661 Another example with mid-level reliability, that was randomly selected from the mappings, was 662 the association between the HPO term "anemic pallor" (HP:0001017) and the CC category GO 663 term "Fanconi anaemia nuclear complex" (GO:0043240). The exact definition of this GO term in 664 the UniProt-GOA database is: "A protein complex composed of the Fanconi anaemia (FA) proteins 665 including $A, C, E, G$ and $F(F A N C A-F)$. Functions in the activation of the downstream protein 666 FANCD2 by monoubiquitylation, and is essential for protection against chromosome breakage." 667 (URL: https://www.ebi.ac.uk/QuickGO/term/GO:0043240). The textual definition of the 668 corresponding HPO term (HP:0001017) is "A type of pallor that is secondary to the presence of 669 anemia". These two terms were mapped to each other in HPO2GO with mid-level confidence (i.e., $670 S=0.64$ and $n=8$ ). The symbols of the co-annotated genes were FANCA, FANCB, FANCC, 671 FANCE, FANCF, FANCG, FANCL and FANCM. These genes are parts of the Fanconi anaemia 672 (FA) protein complex, which protects the cell against chromosomal breakage (Pace et al., 2002). 673 The corresponding CC GO term (GO:0043240) directly describes the FA complex; whereas, the 674 mapped HPO term (HP:0001017) is associated with the Fanconi anemia disease sub-types in the 675 HPO database (e.g., OMIM:600901, OMIM:227650, OMIM:227645, OMIM:227646). According 676 to the disease definition in OMIM, Fanconi anemia is a heterogeneous disorder associated with 677 genomic instability, mainly characterized by developmental abnormalities in major organ systems 678 (Deakyne and Mazin, 2011). Each of the different sub-types listed in OMIM is associated with a 679 mutation in a different FA complex gene. This is a clear example for a HPO term defining an 680 abnormal condition, that is caused by the disease rooted from the disfunction in the biomolecular 681 complex defined by the mapped GO term.

682 An example with low-level reliability (parameter values: $0.2>S>0.1$ and $n=2$ ) is the case where 683 "abnormality of reproductive system physiology" (HP:0000080) HPO term was associated with 684 "collagen catabolic process" (GO:0030574) BP GO term. The parameter values for this mapping 685 were $S=0.11$ and $n=2$. The symbols of the co-annotated genes were COL7A1 and MMP1. The 686 definition of this GO term in the UniProt-GOA database is: "The proteolytic chemical reactions 
687 and pathways resulting in the breakdown of collagen in the extracellular matrix, usually carried 688 out by proteases secreted by nearby cells." (URL: 689 https://www.ebi.ac.uk/QuickGO/term/GO:0030574). On the other hand, HP:0000080 is a generic 690 phenotype term that has 45 descendent terms, and it is possible to reach the root of the sub-ontology 691 from this term in just 3 term-to-term jumps. Naturally, HP:0000080 has been associated with high 692 number of diseases (i.e., 465) in the HPO database, one of which is the Myotonic dystrophy 693 (OMIM:160900). Myotonic dystrophy is an autosomal disorder characterized by muscular 694 dystrophy, myotonia, hypogonadism (i.e., functional activity related issues in the testes/ovaries), 695 and etc. (Musova et al., 2009). In this sense, the relationship between muscular dystrophy and the 696 collagen breakdown is evident; as a result, it can be stated that HPO2GO identified a relevant 697 mapping. However, HP:0000080 is quite generic and associated with many other processes besides 698 muscular dystrophy; consequently, the target mapping is not very specific.

699 The last randomly selected case study example is another one from the low-level reliability bin. 700 The HPO term "polycythemia" (HP:0001901) and the BP category GO term "bicarbonate 701 transport" (GO:0015701) were mapped to each other with $S=0.15$ and $n=2$. The symbols of the 702 co-annotated genes were $H B A 1$ and $H B B$ (i.e., haemoglobin sub-units). The definition of 703 GO:0015701 in the UniProt-GOA database is "The directed movement of bicarbonate into, out of 704 or within a cell, or between cells, by means of some agent such as a transporter or pore" (URL: 705 https://www.ebi.ac.uk/QuickGO/term/GO:0015701). One of the ways the carbon dioxide is 706 removed from tissues is first the generation of carbonic acid, and then the decomposition of

707 708 709

710

711

712

713

714

715

716

717 carbonic acid into bicarbonate to be transported to the lungs, via the red blood cells (i.e., erythrocytes). This process also drives the transport of oxygen molecules from blood to the tissues via an allosteric mechanism. Thus, bicarbonate transport process have high importance for oxygenation. The definition of HP:0001901 in the HPO database is "Polycythemia is diagnosed if the red blood cell count, the haemoglobin level, and the red blood cell volume all exceed the upper limits of normal". HP:0001901 is also associated with 23 diseases most of which are blood related. A few of these diseases are polycythemia vera - PV (OMIM:263300) and various sub-types of familial erythrocytosis (OMIM:13310, OMIM:263400, OMIM:609820, OMIM:611783). Erythrocytosis is an autosomal disorder characterized by increased haemoglobin concentration, increased mass of serum red blood cells, and etc. (Kralovics et al., 1998). It is probable that certain mutations in the proteins that take part in the bicarbonate transport process would cause a 
718 disruption in this function, which would in turn lead to decreased efficiency in both the 719 oxygenation of tissues, and the removal of carbon dioxide from them, causing the polycythemia 720 phenotype related diseases such as polycythemia vera or erythrocytosis. In this example, HPO2GO 721 managed to return an indirect but relevant association.

722 


\section{4. DISCUSSION}

724 HPO project's manual HPO-GO term associations have been generated only for a sub-set of HPO 725 and GO terms, by comparing the term definitions. As a result, the coverage of these associations 726 is limited (i.e., a total of 489 mappings). In HPO2GO approach, all GO-HPO term combinations

727 that satisfy the co-occurrence similarity test conditions were linked. This way, the non-documented 728 relations were also identified. The results of the mapping comparison analysis has indicated that 729 the direct correspondence between the manual associations and HPO2GO was low, mainly due to 730 the limitations in the source annotation sets. Nearly all of the manual HPO-GO associations had 731 highly similar definitions. Whereas, HPO2GO retrieved associations with mostly dissimilar term 732 definitions, which often made biological sense considering the underlying molecular mechanisms.

733 The manual identification of this type of associations may require a comprehensive curation 734 process. In this context, it is expected that the HPO2GO mappings will be valuable for the research 735 community.

736 In this study, individual terms from both ontologies were mapped to each other considering the 737 co-annotated genes/proteins. However, the initial design of the experiment considered the mapping 738 of an HPO term to a trio of GO terms, one from each GO category (i.e., biological process - BP, 739 molecular function - MF and cellular component - CC). This way, the corresponding phenotypic 740 abnormality would be associated with a problem in a specific molecular event (defined by the MF 741 term), as a part of a defined large-scale process (the BP term), occurring at a particular sub-cellular 742 location (the $\mathrm{CC}$ term). This approach would have been biologically more relevant compared to

743 the current design; however, the initial design failed due to the scarcity of both HPO annotations 744 and MF, BP and CC GO term containing triple annotations. At this point in the study, a second 745 option was considered, where HPO terms were tried to be mapped to MF and BP term pairs; 746 nevertheless, the same problem was encountered again. Reliable annotation sets with higher 747 coverage, which may become available in the future with more curation efforts, may solve this 748 problem and make the abovementioned mapping approach practical. However today, even for the 749 currently applied one to one term mapping approach, the main challenge is the low coverage of 750 the predicted associations due to the small size of the source annotation sets. There can be a few 751 alternative solutions to this problem. First of all, the training sets with enriched GO annotation 752 may be obtained by including the annotations with evidence codes of reduced reliability (e.g., IEA 753 - electronically generated), as this approach has been shown to work well with the CAFA2 
754 benchmark set. Another option for enlarging the GO annotations would be utilizing the 755 gene/protein similarity information (i.e., protein function prediction). Scaling up the coverage of 756 both the HPO and the GO sets can be provided by propagating the annotations to the parent terms 757 according to the true path rule. "HPO2GO asserted vs. propagated mappings" analysis have 758 indicated that this approach significantly increases the coverage. Another option here would be 759 assuming a more elaborate approach in the mapping procedure by taking the graph-based 760 hierarchical term relationships into account while generating the HPO2GO mappings (i.e., the 761 parent and child terms of the target HPO-GO term pair, that are co-annotated to different 762 genes/proteins, will also contribute to the calculation of the co-occurrence similarity of the target

763 764

765

766

767

768

769

770

771

772

773

774

775

776

777

778

779

780

781

782

783

784 HPO-GO pair). This approach have been widely accepted in the area of sematic similarity based functional analysis of biomolecules.

The official CAFA2 challenge results have indicated that, the methods based on sequence similarities (e.g., the baseline classifier BLAST and a few models from the participating groups) can achieve a good predictive performance considering the GO terms in the molecular function (MF) category. This was expected since it is possible to detect most of the signatures related to the molecular functions by analysing the amino acid sequence. However, most of the sequencesimilarity based methods failed in predicting the cellular component (CC) GO term and HPO term associations. This can be explained for $\mathrm{CC}$ terms as either by the cleavage of the signals from the sequence post-translationally or the difficulties in detecting weak signals used for directing proteins to different compartments. Considering the HPO prediction, the case may completely be different. As opposed to GO terms, which define the attributes the proteins contain, HPO terms define phenotypic abnormalities caused by the protein when it loses one (or more) of its functions, usually due to certain mutations in the gene that codes the protein. Due to this reason, transferring a HPO annotation from one protein to another based on sequence similarity does not have a biological relevance, which explains the poor performance of the BLAST classifier.

It was interesting to see that the HPO2GO CAFA2 benchmark set predictions produced using the training set of asserted annotations resulted in the same overall performance as the predictions produced using the training set of propagated annotations (without the inclusion of electronically made GO annotations). In theory, employing the true path rule propagation would enrich the training set (i.e., less false negatives) without any sacrifice in terms of the type I error (i.e., the same number of false positives), which should have produced an elevated Fmax value. Instead, the 
785 performance remained the same. There could be two possible reasons for this. First, there were 786 already false positive instances in the training set beforehand, and the propagation process just 787 made the situation worse by increasing the number of false positive instances in a magnitude equal 788 to the number of ancestor terms of the false positive instances (i.e., equal to the number of 789 propagation operations). This in turn compensated for the increase in the coverage obtained by 790 propagating the annotations. The second reason could be that, there were no significant errors 791 regarding the propagated HPO2GO predictions; however, the benchmark annotations were 792 incomplete. As a result, a portion of the real true positive predictions were counted as false 793 positives. Nevertheless, employing the propagated HPO annotations together with the enlarged 794 GO annotation set (including the electronically made annotations) have increased the predictive 795 performance by nearly $17 \%$. However, it is not possible to be sure about the accuracy of these 796 results due to the small size of the CAFA2 test dataset. A larger benchmark annotation set that is 797 guaranteed to be complete would be required in order to discuss the performance further.

798 An important observation regarding the CAFA tests done in this study is that, there was a large 799 difference between the number of HPO predictions for CAFA2 and CAFA3 targets, using 800 HPO2GO with default parameters (i.e., 1,922,333 in CAFA2 as opposed to 3,453,130 in CAFA3).

801 There was also an increase in the number of predicted HPO terms (i.e., 2,640 in CAFA2 as opposed 802 to 3,719 in CAFA3), and there were no significant increase in the number of targets. The increase 803 in the number of predictions and the predicted HPO terms can be attributed to the training set 804 getting larger and more informative in time. The training set used for CAFA2 contained 133,175 805 annotations; whereas, it was 153,575 for CAFA3. The comparison of the predictive performances 806 of HPO2GO trained by the CAFA2 and the CAFA3 training sets may reveal more about the 807 situation.

808 Considering the HPO2GO biological relevance case studies, it was possible to find a biological 809 connection between the mapped HPO and GO terms in most cases; however, the connection 810 became indirect and more abstract, when the $S$ and $n$ values were low (i.e., reduced reliability). 811 Frequently, highly generic/shallow HPO and GO terms were mapped to multiple terms from the 812 other ontology, with generally low parameter values. These mappings were still observed to be 813 relevant but less informative. On the other hand, most of the mappings with high-level reliability 814 were between specific HPO and GO terms, and the selected cases from these mappings were 815 observed to be highly informative. Similar to the other automated methods that produce predictions 
816 on the biological data, HPO2GO has limitations. This was also reflected in the results of the 817 predictive performance test on the CAFA2 benchmark set (Fmax $=0.35)$, which can be considered 818 low for real-life applications. For HPO2GO to be employed in biological data analysis pipelines 819 in the future, HPO-GO term mappings should also be manually curated.

820 


\section{5. CONCLUSION}

822 In this study, a simple and effective strategy, HPO2GO, was proposed to semantically map 823 phenotypic abnormality defining HPO terms with biomolecular function defining GO terms, 824 considering the cross-ontology annotation co-occurrences on different genes/proteins. This 825 approach can easily be translated into novel HPO term predictions for genes/proteins. A literature 826 based case study was carried to discuss the biological relevance of the selected HPO2GO 827 mappings. This work also presents an application of the cross-ontology term mapping approach 828 by generating HPO-protein associations. HPO2GO was benchmarked on CAFA2 challenge 829 protein targets and it was revealed that the method was among the best performers of the HPO 830 term prediction track participators (i.e., the state-of-the-art methods). Also, the up-to-date trained 831 system was employed to predict HPO associations for all human proteins in the UniProtKB/Swiss-

832 Prot database (i.e., HPO2protein predictions). The methodology proposed here may also support 833 the already established approaches (e.g., text mining), as it is possible for different techniques with 834 different data sources and perspectives to produce results that complement distinct missing pieces 835 of the knowledge space. This property is often utilized in ensemble based classification 836 approaches. In this sense, It would also be interesting to analyse the complementarity between the 837 predictions of the proposed method and the predictions of the state-of-the-art approaches 838 participated in CAFA2 challenge; however, this was not possible since the actual prediction results 839 of the participant groups are not publicly available.

840 As for the future work, it is first planned to map the HPO terms to GO term trios (i.e., MF, BP and $841 \mathrm{CC}$ terms at the same time) using enriched annotation datasets, as explained at the discussion 842 section. Another future task is the integration of HPO2GO mappings to our freely available GO 843 based automated protein function prediction tool/server UniGOPred (Rifaioglu et al., 2018); so 844 that, query proteins that receive a GO term prediction will be automatically associated with the 845 HPO term(s) that are mapped to the corresponding GO term. It is expected that this approach 846 would produce large-scale HPO predictions for uncharacterized proteins without any curated 847 annotation, where the only available information is the amino acid sequence. The knowledge 848 extraction methodology proposed here can easily be combined with various types of protein 849 features employed in other predictive methods (e.g., variant information, PPIs, gene expression 850 profiles, etc.) to generate an ensemble HPO term prediction tool that identifies novel HPO851 gene/protein-disease associations. 


\section{6. REFERENCES}

853 Alvarez MA, Qi X, Yan C. 2011. A shortest-path graph kernel for estimating gene product 854 semantic similarity. Journal of biomedical semantics, 2(1), 3.

855 Amberger JS, Bocchini CA, Schiettecatte F, Scott AF, Hamosh A. 2014. OMIM. org: Online 856 Mendelian Inheritance in Man (OMIM $®)$, an online catalog of human genes and genetic disorders. 857 Nucleic acids research, 43(D1), D789-D798.

858 Barth PG. 2014. Pontocerebellar hypoplasias: an overview of a group of inherited 859 neurodegenerative disorders with fetal onset. Brain Dev., 15: 411-422.

860 Bromberg Y. 2013. Disease gene prioritization. PLoS computational biology, 9(4), e1002902.

861 Budde BS, Namavar Y, Barth PG, Poll-The BT, Nürnberg G, Becker C, van Ruissen F, Weterman 862 MA, Fluiter K, te Beek ET, Aronica E, van der Knaap MS, Höhne W, Toliat MR, Crow YJ, 863 Steinling M, Voit T, Roelenso F, Brussel W, Brockmann K, Kyllerman M, Boltshauser E, 864 Hammersen G, Willemsen M, Basel-Vanagaite L, Krägeloh-Mann I, de Vries LS, Sztriha L, 865 Muntoni F, Ferrie CD, Battini R, Hennekam RC, Grillo E, Beemer FA, Stoets LM, Wollnik B, 866 Nürnberg P, Baas F. 2008. tRNA splicing endonuclease mutations cause pontocerebellar 867 hypoplasia. Nature genetics, 40(9), 1113.

868 Clark, WT, Radivojac, P. 2013. Information-theoretic evaluation of predicted ontological 869 annotations. Bioinformatics, 29(13), i53-i61.

870 Cozzetto D, Buchan DW, Bryson K, Jones DT. 2013. Protein function prediction by massive 871 integration of evolutionary analyses and multiple data sources. BMC bioinformatics, 14(3), p.S1.

872 Deakyne JS, Mazin AV. (2011). Fanconi anemia: at the crossroads of DNA repair. Biochemistry 873 (Moscow), 76(1), 36-48.

874 Deng Y, Gao L, Wang B, Guo X. 2015. HPOSim: an R package for phenotypic similarity measure 875 and enrichment analysis based on the human phenotype ontology. PloS one, 10(2), e0115692.

876 Doğan T, MacDougall A, Saidi R, Poggioli D, Bateman A, O’Donovan C, Martin MJ. 2016. 877 UniProt-DAAC: domain architecture alignment and classification, a new method for automatic 878 functional annotation in UniProtKB. Bioinformatics, 32(15), 2264-2271.

879 Falda M, Toppo S, Pescarolo A, Lavezzo E, Di Camillo B, Facchinetti A, Cilia E, Velasco R, 
880 Fontana P. 2012. Argot2: a large scale function prediction tool relying on semantic similarity of 881 weighted Gene Ontology terms. BMC bioinformatics, 13(4), S14.

882 Fang H, Gough J. 2012. DcGO: database of domain-centric ontologies on functions, phenotypes, 883 diseases and more. Nucleic acids research, 41(D1), D536-D544.

884 Finn RD, Attwood TK, Babbitt PC, Bateman A, Bork P, Bridge AJ, Chang HY, Dosztányi Z, El885 Gebali S, Fraser M, Gough J, Haft D, Holliday GL, Huang H, Huang X, Letunic I, Lopez R, Lu S, 886 Marchler-Bauer A, Mi H, Mistry J, Natale DA, Necci M, Nuka G, Orengo CA, Park Y, Pesseat S, 887 Piovesan D, Potter SC, Rawlings ND, Redaschi N, Richardson L, Rivoire C, Sangrador-Vegas A, 888 Sigrist C, Sillitoe I, Smithers B, Squizzato S, Sutton G, Thanki N, Thomas PD, Tosatto SC, Wu 889 CH, Xenarios I, Yeh LS, Young SY, Mitchell AL. 2016. InterPro in 2017-beyond protein family 890 and domain annotations. Nucleic acids research, 45(D1), D190-D199.

891 Firth HV, Richards SM, Bevan AP, Clayton S, Corpas M, Rajan D, Van Vooren S, Moreau Y, 892 Pettett RM, Carter NP. 2009. DECIPHER: database of chromosomal imbalance and phenotype in 893 humans using ensembl resources. The American Journal of Human Genetics, 84(4), 524-533.

894 Gene Ontology Consortium. 2014. Gene ontology consortium: going forward. Nucleic acids 895 research, 43(D1), D1049-D1056.

896 Groza T, Köhler S, Moldenhauer D, Vasilevsky N, Baynam G, Zemojtel T, Schriml LM, Kibbe 897 WA, Schofield PN, Beck T, Vasant D, Brookes AJ, Zankl A, Washington NL, Mungall CJ, Lewis 898 SE, Haendel MA, Parkinson H, Robinson PN. 2015. The human phenotype ontology: semantic 899 unification of common and rare disease. The American Journal of Human Genetics, 97(1), 111900124.

901 Guala D, Sonnhammer EL. 2017. A large-scale benchmark of gene prioritization methods. 902 Scientific reports, 7, 46598.

903 Guney E, Oliva B. 2014. Analysis of the robustness of network-based disease-gene prioritization 904 methods reveals redundancy in the human interactome and functional diversity of disease-genes. 905 PLoS one, 9(4), e94686.

906 Hawkins T, Chitale M, Luban S, Kihara D. 2009. PFP: Automated prediction of gene ontology 907 functional annotations with confidence scores using protein sequence data. Proteins: Structure, 908 Function, and Bioinformatics, 74(3), 566-582. 
909 Hollander M, Wolfe DA, Chicken E. 2013. Nonparametric statistical methods. John Wiley \& 910 Sons.

911 Huntley RP, Sawford T, Mutowo-Meullenet P, Shypitsyna A, Bonilla C, Martin MJ, O'Donovan 912 C. 2015. The GOA database: gene ontology annotation updates for 2015. Nucleic acids research, 913 43(D1):1057-63.

914 Jiang Y, Oron TR, Clark WT, Bankapur AR, D'Andrea D, Lepore R, Funk CS, Kahanda I, 915 Verspoor KM,, Ben-Hur A, Koo da CE, Penfold-Brown D,, Shasha D, Youngs N,,, Bonneau R,, 916 Lin A, Sahraeian SM, Martelli PL, Profiti G, Casadio R, Cao R, Zhong Z, Cheng J, Altenhoff A, 917 Skunca N,, Dessimoz C,, Dogan T, Hakala K,, Kaewphan S,,, Mehryary F,, Salakoski T,, Ginter 918 F, Fang H, Smithers B, Oates M, Gough J, Törönen P, Koskinen P, Holm L, Chen CT, Hsu WL, 919 Bryson K, Cozzetto D, Minneci F, Jones DT, Chapman S, Bkc D, Khan IK, Kihara D,, Ofer D, 920 Rappoport N,, Stern A,, Cibrian-Uhalte E, Denny P, Foulger RE, Hieta R, Legge D, Lovering RC, 921 Magrane M, Melidoni AN, Mutowo-Meullenet P, Pichler K, Shypitsyna A, Li B, Zakeri P, ElShal 922 S, Tranchevent LC, , Das S, Dawson NL, Lee D, Lees JG, Sillitoe I, Bhat P, Nepusz T, Romero 923 AE, Sasidharan R, Yang H, Paccanaro A, Gillis J, Sedeño-Cortés AE, Pavlidis P, Feng S, Cejuela 924 JM, Goldberg T, Hamp T, Richter L, Salamov A, Gabaldon T,,, Marcet-Houben M,, Supek F,, 925 Gong Q, Ning W, Zhou Y, Tian W, Falda M, Fontana P, Lavezzo E, Toppo S, Ferrari C, Giollo 926 M, Piovesan D, Tosatto SC, Del Pozo A, Fernández JM, Maietta P, Valencia A, Tress ML, Benso 927 A, Di Carlo S, Politano G, Savino A, Rehman HU, Re M, Mesiti M, Valentini G, Bargsten JW, 928 van Dijk AD,, Gemovic B, Glisic S, Perovic V, Veljkovic V, Veljkovic N, Almeida-E-Silva DC, 929 Vencio RZ, Sharan M, Vogel J, Kansakar L, Zhang S, Vucetic S, Wang Z, Sternberg MJ, Wass 930 MN, Huntley RP, Martin MJ, O'Donovan C, Robinson PN, Moreau Y, Tramontano A, Babbitt PC, 931 Brenner SE, Linial M, Orengo CA, Rost B, Greene CS, Mooney SD, Friedberg I, Radivojac P. 932 2016. An expanded evaluation of protein function prediction methods shows an improvement in 933 accuracy. Genome biology, 17(1), 184.

934 Kahanda I, Funk C, Verspoor K, Ben-Hur A. 2015. PHENOstruct: Prediction of human phenotype 935 ontology terms using heterogeneous data sources. F1000Research, 4.

936 Kibbe WA, Arze C, Felix V, Mitraka E, Bolton E, Fu G, Mungall CJ, Binder JX, Malone J, Vasant 937 D, Parkinson H, Schriml LM . 2014. Disease ontology 2015 update: an expanded and updated 938 database of human diseases for linking biomedical knowledge through disease data. Nucleic acids 
939 research, 43:1071-8.

940 Köhler S, Schulz MH, Krawitz P, Bauer S, Dölken S, Ott CE, Mundlos C, Horn D, Mundlos S, 941 Robinson PN. 2009. Clinical diagnostics in human genetics with semantic similarity searches in 942 ontologies. The American Journal of Human Genetics, 85(4), 457-464.

943 Köhler S, Vasilevsky NA, Engelstad M, Foster E, McMurry J, Aymé S, Baynam G,, Bello SM, 944 Boerkoel CF, Boycott KM, Brudno M, Buske OJ, Chinnery PF, Cipriani V,, Connell LE, Dawkins 945 HJ, DeMare LE, Devereau AD, de Vries BB, Firth HV, Freson K, Greene D, Hamosh A, Helbig 946 I,, Hum C, Jähn JA, James R,, Krause R, F Laulederkind SJ, Lochmüller H, Lyon GJ, Ogishima 947 S, Olry A, Ouwehand WH, Pontikos N,, Rath A, Schaefer F, Scott RH, Segal M, Sergouniotis PI, 948 Sever R, Smith CL, Straub V, Thompson R, Turner C, Turro E,, Veltman MW, Vulliamy T, Yu J, 949 von Ziegenweidt J, Zankl A, Züchner S, Zemojtel T, Jacobsen JO, Groza T, Smedley D, Mungall 950 CJ, Haendel M, Robinson PN. 2016. The human phenotype ontology in 2017. Nucleic acids 951 research, 45(D1), D865-D876.

952 Krallinger M, Valencia A, Hirschman L. 2008. Linking genes to literature: text mining, 953 information extraction, and retrieval applications for biology. Genome biology, 9(2), S8.

954 Kralovics R, Sokol L, Prchal, JT. 1998. Absence of polycythemia in a child with a unique 955 erythropoietin receptor mutation in a family with autosomal dominant primary polycythemia. The 956 Journal of clinical investigation, 102(1), 124-129.

957 Kulmanov M, Hoehndorf R. 2017. Evaluating the effect of annotation size on measures of 958 semantic similarity. Journal of biomedical semantics, 8(1), 7.

959 Lan L, Djuric N, Guo Y, Vucetic S. 2013. MS-k NN: protein function prediction by integrating 960 multiple data sources. BMC bioinformatics, 14(3), p.S8.

961 Lilliefors HW. 1967. On the Kolmogorov-Smirnov test for normality with mean and variance 962 unknown. Journal of the American statistical Association, 62(318), 399-402.

963 Lin D. 1998. An information-theoretic definition of similarity. Proc. of the 15th International 964 Conference on Machine Learning. San Francisco, CA: Morgan Kaufmann. pp. 296-304.

965 Moreau Y, Tranchevent LC. 2012. Computational tools for prioritizing candidate genes: boosting 966 disease gene discovery. Nature reviews genetics, 13(8), 523. 
967 Musova Z, Mazanec R, Krepelova A, Ehler E, Vales J, Jaklova R, Prochazka T, Koukal P, 968 Marikova T, Kraus J, Havlovicova M, Sedlacek Z. 2009. Highly unstable sequence interruptions 969 of the CTG repeat in the myotonic dystrophy gene. American journal of medical genetics Part A, 970 149(7), 1365-1374.

971 Notaro M, Schubach M, Robinson PN, Valentini G. 2017. Prediction of Human Phenotype 972 Ontology terms by means of hierarchical ensemble methods. BMC bioinformatics, 18(1), 449.

973 Pace P, Johnson M, Tan WM, Mosedale G, Sng C, Hoatlin M, de Winter J, Joenje H, Gergely F, 974 Patel KJ. 2002. FANCE: the link between Fanconi anaemia complex assembly and activity. The 975 EMBO journal, 21(13), 3414-3423.

976 Peng J, Li Q, Shang X. 2017. Investigations on factors influencing HPO-based semantic similarity 977 calculation. Journal of biomedical semantics, 8(1), 34.

978 Radivojac P, Clark WT, Oron TR, Schnoes AM, Wittkop T, Sokolov A, Graim K, Funk C, 979 Verspoor K, Ben-Hur A, Pandey G, Yunes JM, Talwalkar AS, Repo S, Souza ML, Piovesan D, 980 Casadio R, Wang Z, Cheng J, Fang H, Gough J, Koskinen P, Törönen P, Nokso-Koivisto J, Holm 981 L, Cozzetto D, Buchan DW, Bryson K, Jones DT, Limaye B, Inamdar H, Datta A, Manjari SK, 982 Joshi R, Chitale M, Kihara D, Lisewski AM, Erdin S, Venner E, Lichtarge O, Rentzsch R, Yang 983 H, Romero AE, Bhat P, Paccanaro A, Hamp T, Kaßner R, Seemayer S, Vicedo E, Schaefer C, 984 Achten D, Auer F, Boehm A, Braun T, Hecht M, Heron M, Hönigschmid P, Hopf TA, Kaufmann 985 S, Kiening M, Krompass D, Landerer C, Mahlich Y, Roos M, Björne J, Salakoski T, Wong A, 986 Shatkay H, Gatzmann F, Sommer I, Wass MN, Sternberg MJ, Škunca N, Supek F, Bošnjak M, 987 Panov P, Džeroski S, Šmuc T, Kourmpetis YA, van Dijk AD, ter Braak CJ, Zhou Y, Gong Q, 988 Dong X, Tian W, Falda M, Fontana P, Lavezzo E, Di Camillo B, Toppo S, Lan L, Djuric N, Guo 989 Y, Vucetic S, Bairoch A, Linial M, Babbitt PC, Brenner SE, Orengo C, Rost B, Mooney SD, 990 Friedberg I. 2013. A large-scale evaluation of computational protein function prediction. Nature 991 methods, 10(3), 221.

992 Rath A, Olry A, Dhombres F, Brandt MMC, Urbero B, Ayme S. 2012. Representation of rare 993 diseases in health information systems: the Orphanet approach to serve a wide range of end users. 994 Hum. Mutat., 33:803-808.

995 Rifaioglu AS, Doğan T, Saraç ÖS, Ersahin T, Saidi R, Atalay MV, Martin MJ, Cetin-Atalay R. 
996 2018. Large-scale automated function prediction of protein sequences and an experimental case 997 study validation on PTEN transcript variants. Proteins: Structure, Function, and Bioinformatics, 998 86(2), 135-151.

999 Robinson PN, Köhler S, Bauer S, Seelow D, Horn D, Mundlos S. 2008. The Human Phenotype 1000 Ontology: a tool for annotating and analyzing human hereditary disease. The American Journal of 1001 Human Genetics, 83(5), 610-615.

1002 Rodríguez-García MÁ, Gkoutos GV, Schofield PN, Hoehndorf R. 2017. Integrating phenotype 1003 ontologies with PhenomeNET. Journal of biomedical semantics, 8(1), 58.

1004 Roy A, Yang J, Zhang Y. 2012. COFACTOR: an accurate comparative algorithm for structure1005 based protein function annotation. Nucleic acids research, 40(W1), W471-W477.

1006 Schlicker A, Lengauer T, Albrecht M. 2010. Improving disease gene prioritization using the 1007 semantic similarity of Gene Ontology terms. Bioinformatics, 26(18), i561-i567.

1008 Smedley D, Oellrich A, Köhler S, Ruef B; Sanger Mouse Genetics Project, Westerfield M, 1009 Robinson P, Lewis S, Mungall C. 2013. PhenoDigm: analyzing curated annotations to associate 1010 animal models with human diseases. Database, bat025.

1011 Smith CL, Goldsmith CAW, Eppig JT. 2005. The Mammalian Phenotype Ontology as a tool for 1012 annotating, analyzing and comparing phenotypic information. Genome biology, 6(1), R7.

1013 UniProt Consortium. 2017. UniProt: the universal protein knowledgebase. Nucleic acids research, 1014 45(D1), D158-D169.

1015 Valentini G, Armano G, Frasca M, Lin J, Mesiti M, Re M. 2016. RANKS: a flexible tool for node 1016 label ranking and classification in biological networks. Bioinformatics, 32(18), 2872-2874.

1017 Van Landeghem S, Björne J, Wei CH, Hakala K, Pyysalo S, Ananiadou S, Kao HY, Lu Z, 1018 Salakoski T, Van de Peer Y, Ginter F. 2013. Large-scale event extraction from literature with 1019 multi-level gene normalization. PloS one, 8(4), e55814.

1020 Vasant D, Chanas L, Malone J, Hanauer M, Olry A, Jupp S, Robinson PN, Parkinson H, Rath A. 1021 2014. Ordo: An ontology connecting rare disease, epidemiology and genetic data. In Proceedings 1022 of ISMB. 
1023 Washington NL, Haendel MA, Mungall CJ, Ashburner M, Westerfield M, Lewis SE. 2009. 1024 Linking human diseases to animal models using ontology-based phenotype annotation. PLoS 1025 biology, 7(11), e1000247.

1026 Wass MN, Barton G, Sternberg MJ. 2012. CombFunc: predicting protein function using 1027 heterogeneous data sources. Nucleic acids research, 40(W1), W466-W470. 


\section{Table $\mathbf{1}$ (on next page)}

Statistics of the initial (i.e., raw) original and randomized HPO-GO mappings $(n \geq 1)$. 
1 Table 1. Statistics of the initial (i.e., raw) original and randomized HPO-GO mappings $(n \geq 1)$.

\begin{tabular}{|c|c|c|c|c|c|c|}
\hline \multirow[b]{2}{*}{$S$} & \multicolumn{2}{|c|}{ \# of mappings } & \multicolumn{2}{|c|}{ \# of mapped HPO terms } & \multicolumn{2}{|c|}{ \# of mapped GO terms } \\
\hline & $\begin{array}{l}\text { Original } \\
\text { mapping }\end{array}$ & $\begin{array}{l}\text { Random } \\
\text { mapping }\end{array}$ & $\begin{array}{l}\text { Original } \\
\text { mapping }\end{array}$ & $\begin{array}{l}\text { Random } \\
\text { mapping }\end{array}$ & $\begin{array}{l}\text { Original } \\
\text { mapping }\end{array}$ & $\begin{array}{l}\text { Random } \\
\text { mapping }\end{array}$ \\
\hline$=1$ & 2433 & 1898 & 844 & 877 & 1108 & 1265 \\
\hline$\geq 0.9$ & 2440 & 1898 & 848 & 877 & 1109 & 1265 \\
\hline$\geq 0.8$ & 2658 & 1899 & 962 & 878 & 1179 & 1266 \\
\hline$\geq 0.7$ & 2805 & 1899 & 1028 & 878 & 1212 & 1266 \\
\hline$\geq 0.6$ & 7355 & 5249 & 1941 & 1653 & 2577 & 2844 \\
\hline$\geq 0.5$ & 8075 & 5252 & 2188 & 1655 & 2712 & 2847 \\
\hline$\geq 0.4$ & 15462 & 9724 & 3014 & 2243 & 4053 & 4207 \\
\hline$\geq 0.3$ & 32393 & 21615 & 4082 & 3017 & 6011 & 6081 \\
\hline$\geq 0.2$ & 63439 & 43593 & 5032 & 3662 & 7569 & 7490 \\
\hline$\geq 0.1$ & 181048 & 134038 & 5920 & 5199 & 8884 & 9005 \\
\hline$>0.0$ & 1433208 & 1543917 & 6005 & 5995 & 9685 & 9685 \\
\hline
\end{tabular}




\section{Table 2 (on next page)}

KS test significance values for the comparison of original vs. randomized distributions

KS test significance values for the comparison of original vs. randomized distributions at different co-occurrence similarity $(S)$ and the number of co-annotated genes $(n)$ thresholds. 
1 Table 2. KS test significance values for the comparison of original vs. randomized distributions at 2 different co-occurrence similarity $(S)$ and the number of co-annotated genes $(n)$ thresholds.

\begin{tabular}{|c|l|l|r|r|r|r|r|r|}
\hline \multirow{2}{*}{ KS test statistic } & \multicolumn{7}{|c|}{ Co-occurrence similarity threshold } \\
\cline { 3 - 9 } \multicolumn{2}{|c|}{} & $\boldsymbol{S}>\mathbf{0}$ & $\boldsymbol{S} \geq \mathbf{0 . 1}$ & $\boldsymbol{S} \geq \mathbf{0 . 2}$ & $\boldsymbol{S} \geq \mathbf{0 . 3}$ & $\boldsymbol{S} \geq \mathbf{0 . 4}$ & $\boldsymbol{S} \geq \mathbf{0 . 5}$ & $\boldsymbol{S} \geq \mathbf{0 . 6}$ \\
\hline \multirow{3}{*}{$\begin{array}{c}\text { \# of co- } \\
\text { annotated } \\
\text { genes } \\
\text { threshold }\end{array}$} & 0.6882 & 0.6884 & 0.4536 & 0.2366 & 0.3921 & 0.3484 & 0.3113 \\
\cline { 2 - 9 } & $\boldsymbol{n} \geq \mathbf{2}$ & 0.0423 & $\mathbf{0 . 0 0 5 7}$ & 0.0005 & 0.0001 & 0.0002 & 0.0038 & $\mathrm{NaN}$ \\
\cline { 2 - 9 } & $\boldsymbol{n} \geq \mathbf{4}$ & 0.2636 & 0.0045 & 0.0000 & $\mathrm{NaN}$ & $\mathrm{NaN}$ & $\mathrm{NaN}$ & $\mathrm{NaN}$ \\
\cline { 2 - 9 } & $\boldsymbol{n} \geq \mathbf{5}$ & 0.3349 & 0.0105 & 0.0000 & $\mathrm{NaN}$ & $\mathrm{NaN}$ & $\mathrm{NaN}$ & $\mathrm{NaN}$ \\
\hline
\end{tabular}

3

4 
Figure 1

Schematic representation of the whole HPO2GO mapping (i.e., training) procedure. 


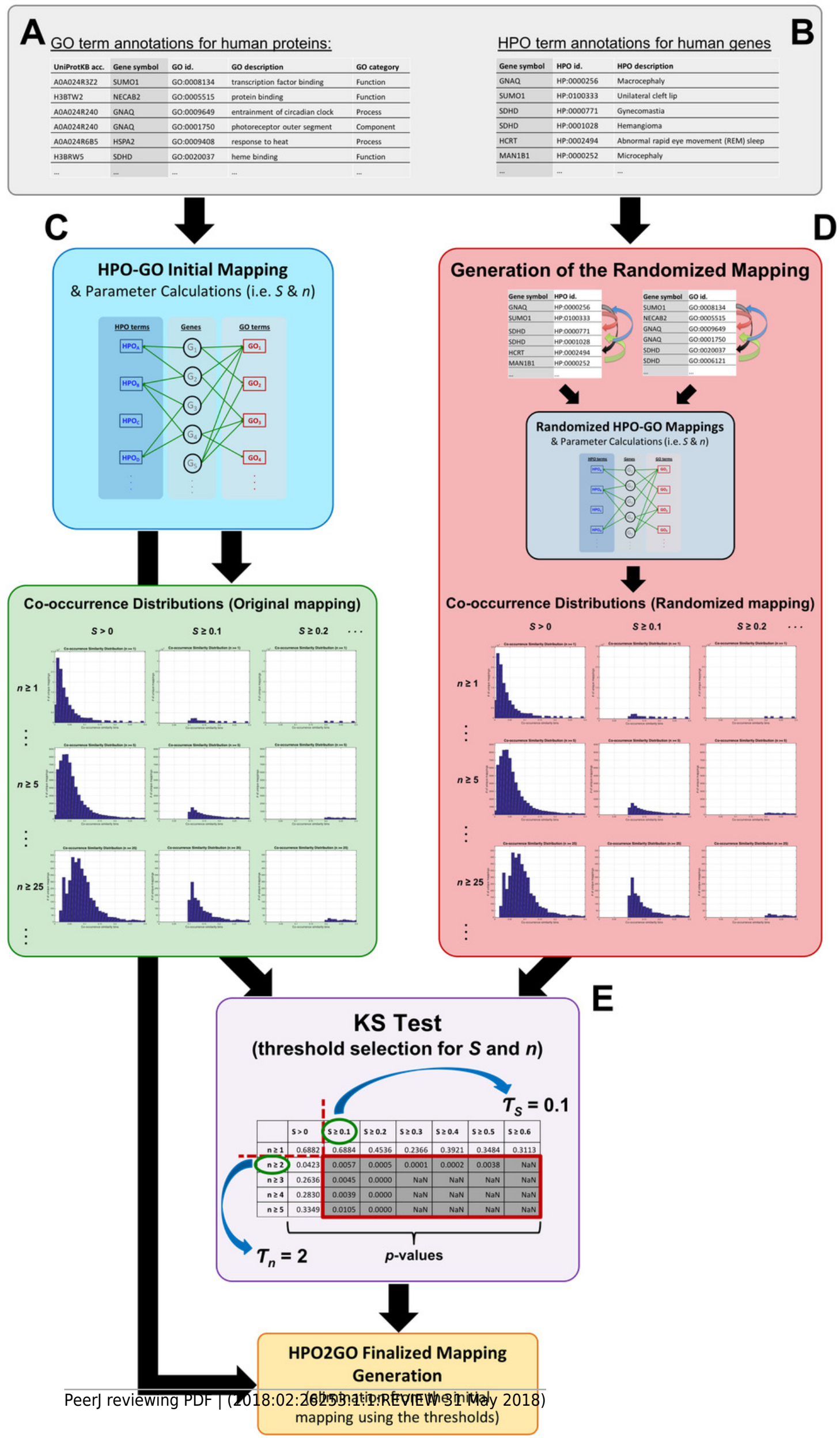


Figure 2

Representation of the initial HPO-GO mapping process

Representation of the initial HPO-GO mapping process together with the calculation of cooccurrence similarities $(S)$ and the number of genes with co-occurring annotations $(n)$, on a toy example.

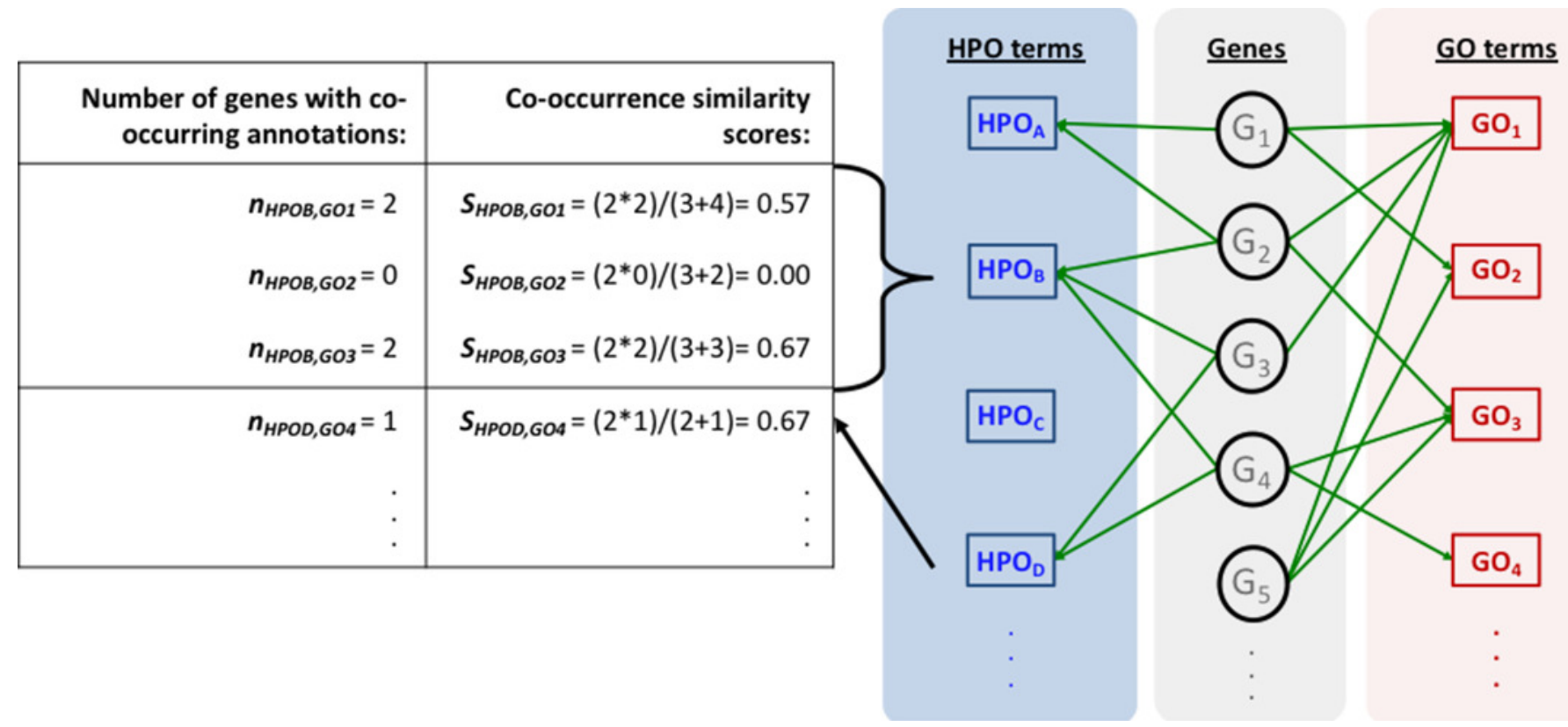


Figure 3

HPO-GO initial mappings co-occurrence similarity distributions

Each plot is drawn for a different value of the number of co-annotated genes (i.e., $n$ ).
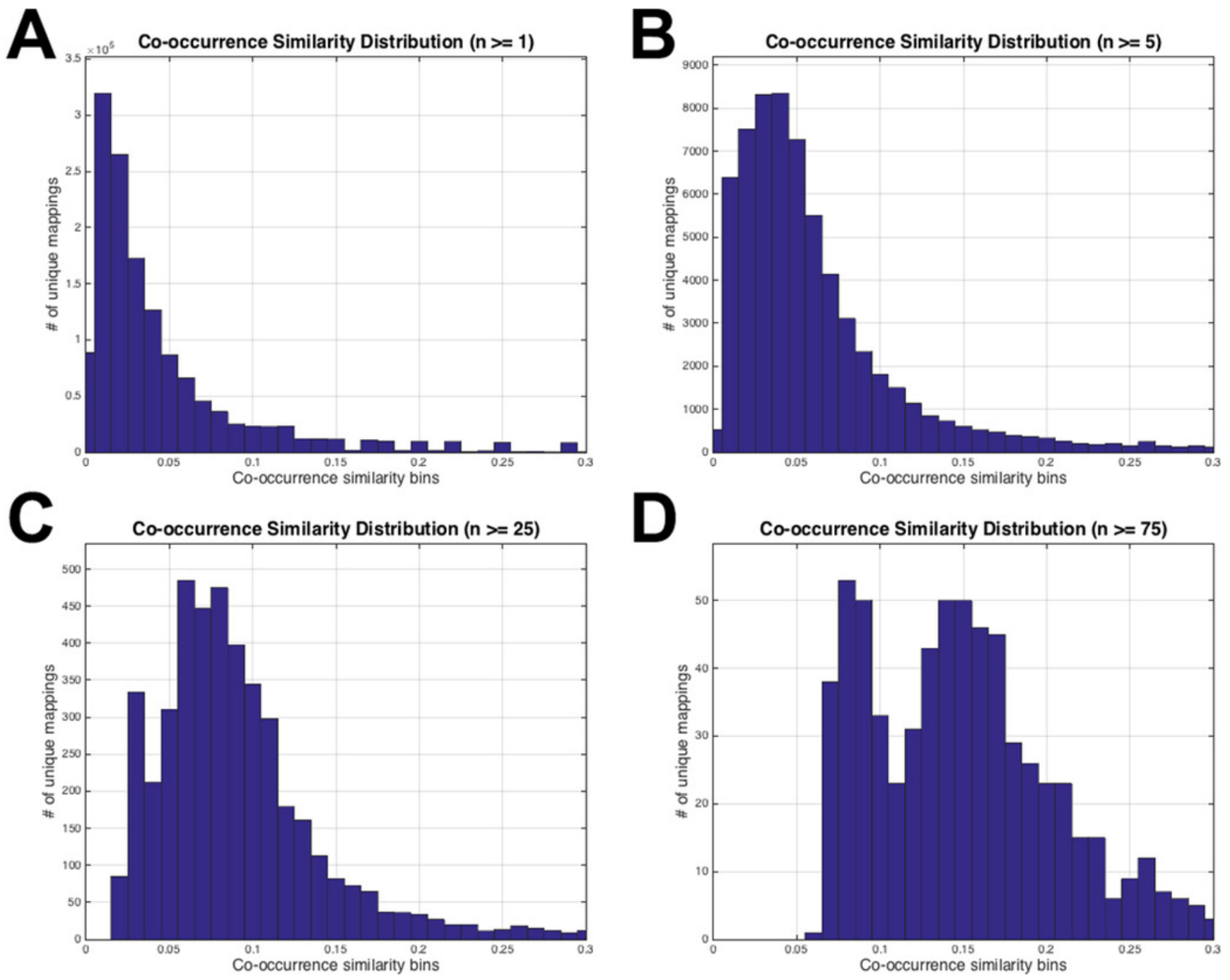


\section{Figure 4}

Cumulative plots displaying the number of HPO-GO mappings for the original (blue curve) and the randomized (red curve) distributions.

Horizontal axis displays the arbitrarily selected co-occurrence similarity thresholds (i.e., $\tau_{s}$ ), and the vertical axis represents the logarithm of the total number of mappings left after the application of the corresponding threshold. Each plot is drawn for a different value of the number of co-annotated genes (i.e., $n$ ). As the threshold (i.e., the minimum required cooccurrence similarity value to keep a mapping in the system) increase, more mappings are eliminated; thus, a monotonic decrease was observed for all plots.

\section{A}

Cumulative \# of Mappings - Original vs. Randomized Distributions (n>=1)

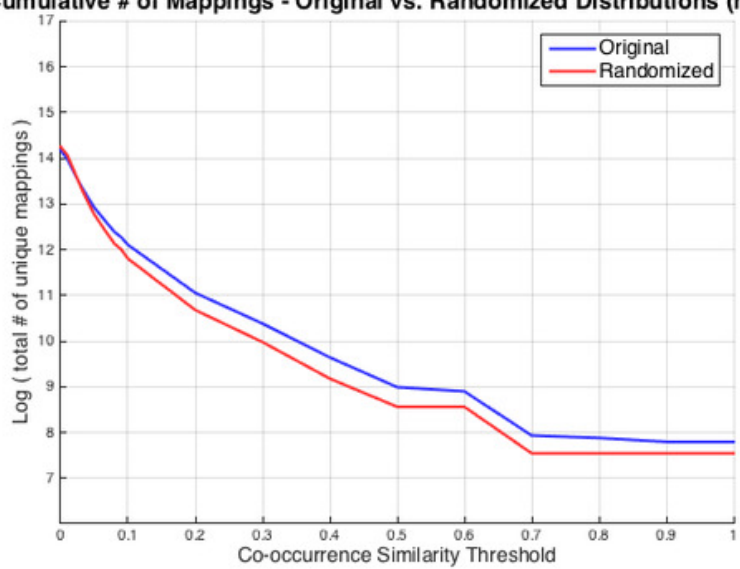

‘

Cumulative \# of Mappings - Original vs. Randomized Distributions ( $>=3$ )

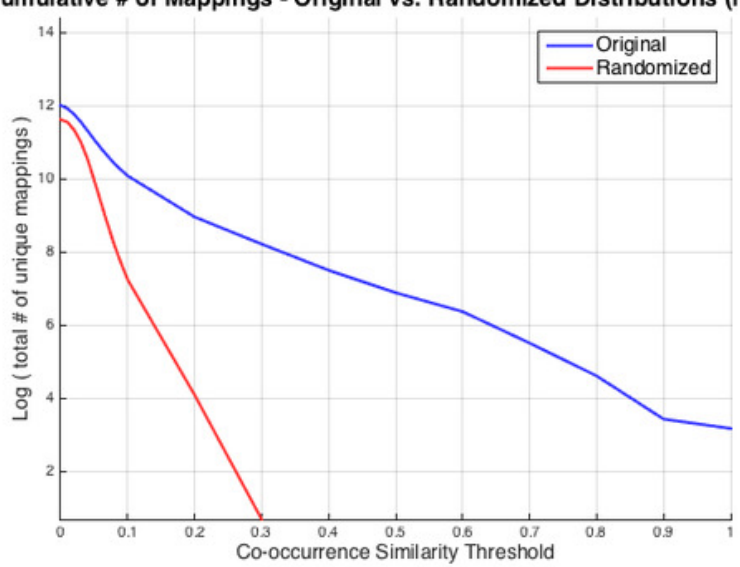

B

Cumulative \# of Mappings - Original vs. Randomized Distributions ( $n>=2$ )

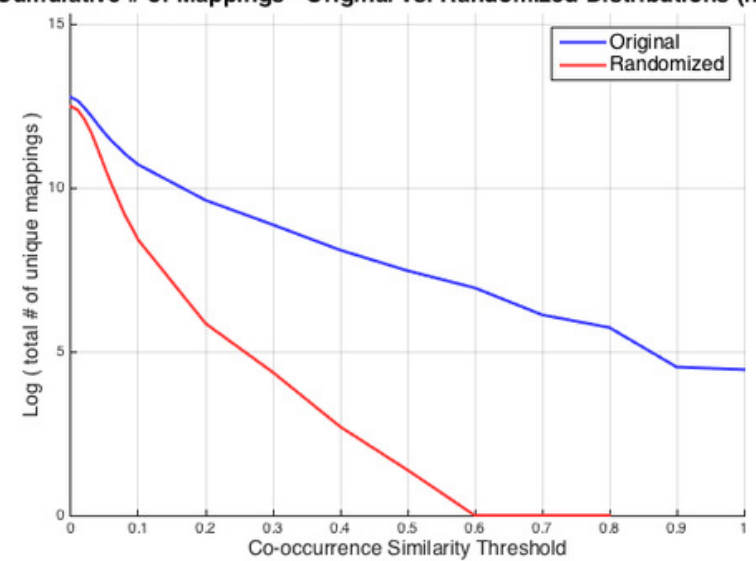

D

Cumulative \# of Mappings - Original vs. Randomized Distributions ( $n>=4$ )

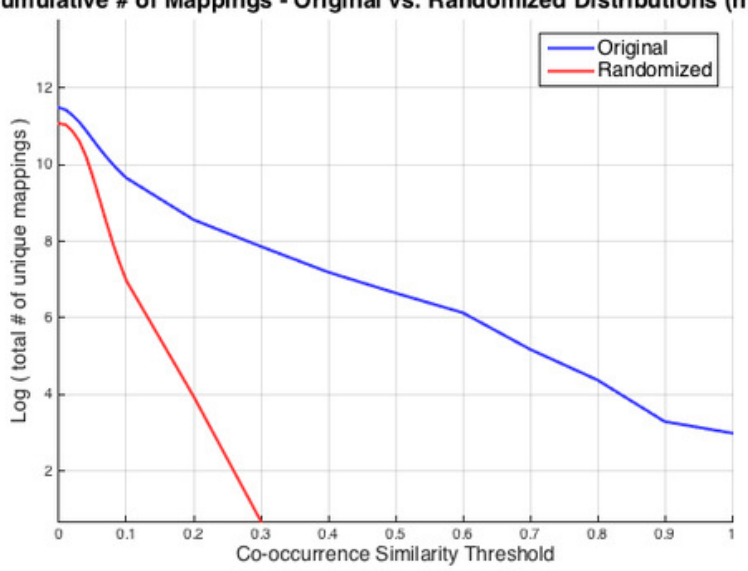


Figure 5

Fmax performance results in CAFA2 HPO prediction benchmark

Performance results (Fmax) of the top performing groups (grey bars), baseline classifiers (red and blue bars) and HPO2GO (dark grey bar) in CAFA2 HPO prediction benchmark. C represents the coverage of predictions on the target protein set.

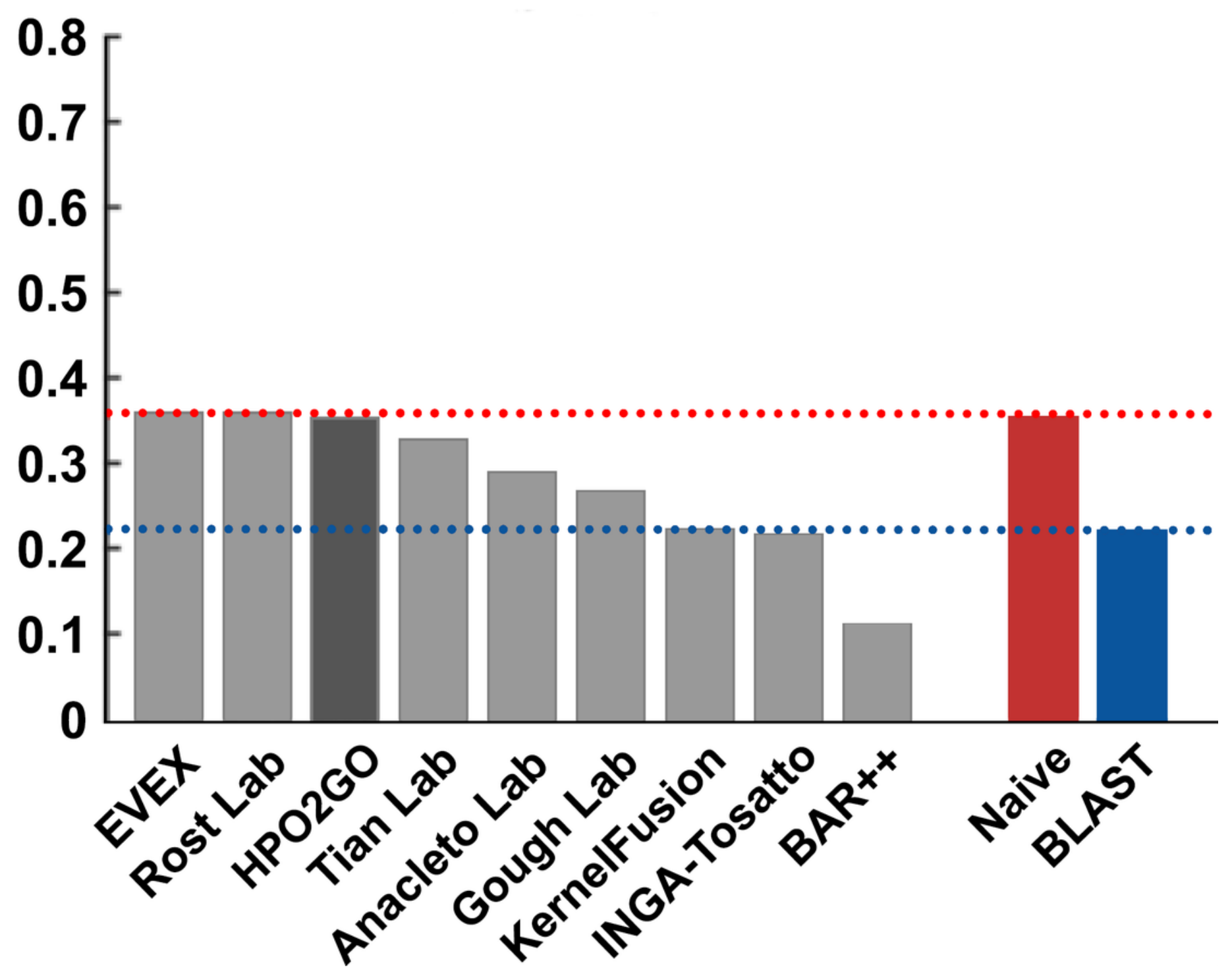




\section{Figure 6}

Precision-recall curves for the CAFA2 HPO prediction benchmark

Precision-recall curves of the top performing groups (coloured curves), baseline classifiers (red and blue dashed curves) and HPO2GO (black curve) in CAFA2 HPO prediction benchmark. The circle on each curve represent the performance at the optimal threshold. Dashed grey iso-performance curves project F-scores. F (inside the box, at the right side) display Fmax values.

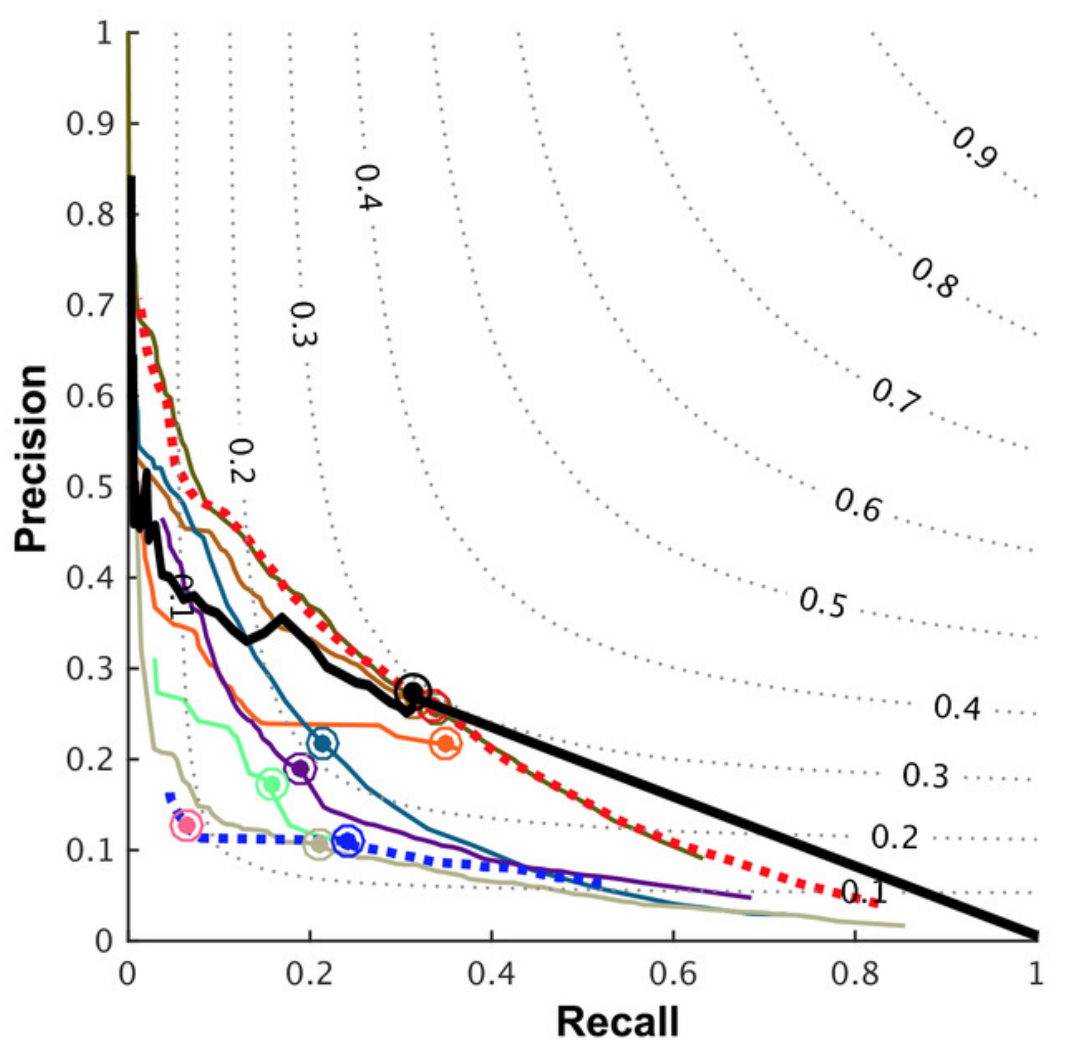

- HPO2GO $(F=0.29, C=0.97)$
Rost Lab $(F=0.29, C=1.00)$
EVEX $(F=0.29, C=1.00)$
Tian Lab $(F=0.27, C=0.97)$
Anacleto Lab $(F=0.22, C=1.00)$
Gough Lab $(F=0.19, C=1.00)$
INGA-Tosatto $(F=0.16, C=0.67)$
KernelFusion $(F=0.14, C=1.00)$
BAR++ $(F=0.09, C=0.25)$
N Naive $(F=0.29, C=1.00)$
BLAST $(F=0.15, C=0.99)$


Figure 7

Normalized remaining uncertainty - misinformation curves for the CAFA2 HPO prediction benchmark

Normalised remaining uncertainty - misinformation curves of the top performing groups (coloured curves), baseline classifiers (red and blue dashed curves) and HPO2GO (black curve) in CAFA2 HPO prediction benchmark. The circle on each curve represent the normalized minimum semantic distance measure (nSmin). Dashed grey iso-performance curves project nSmin values. S (inside the box, at the right side) display nSmin values.

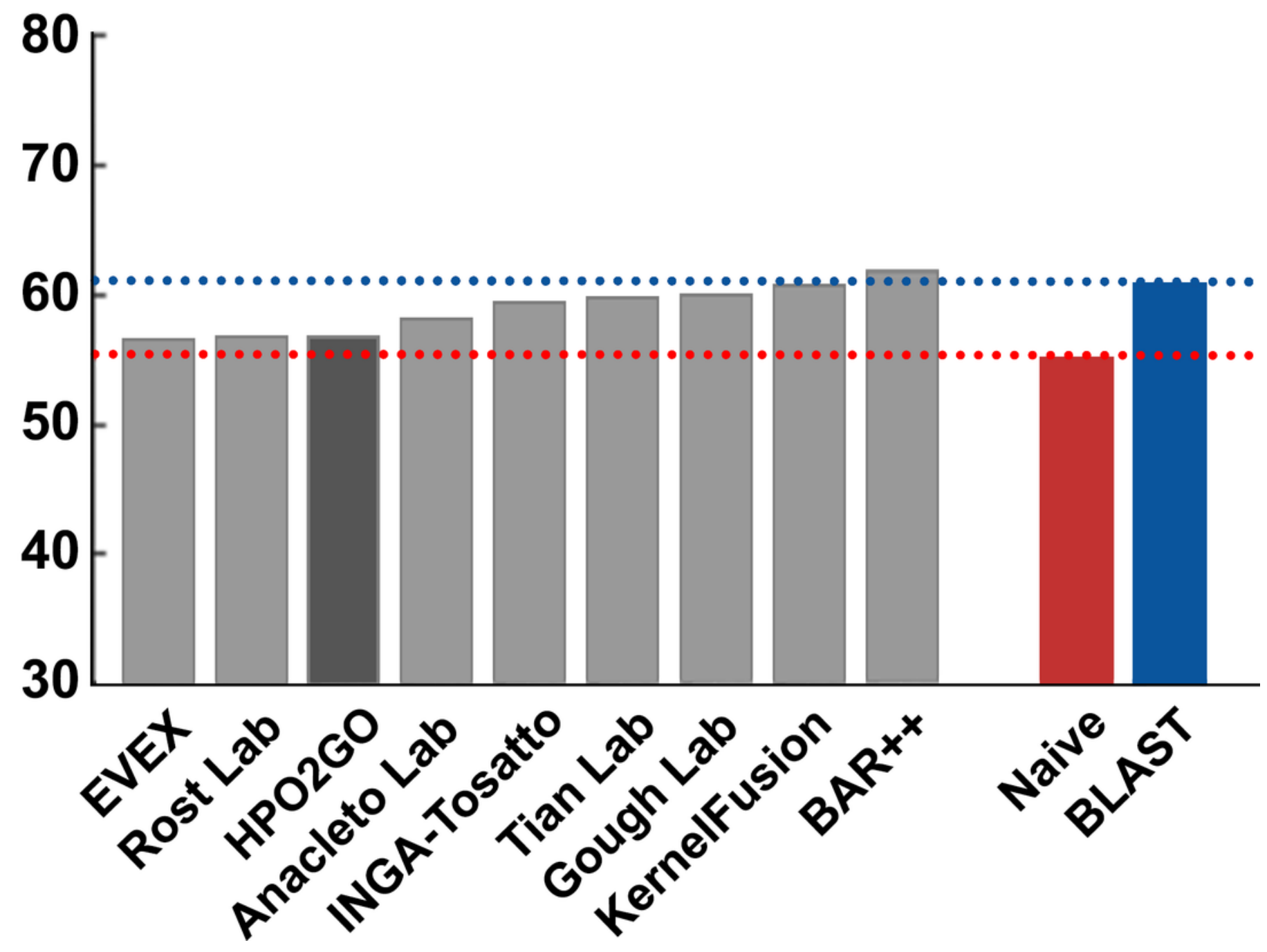


Figure 8

Average term centric AUROC performance results in CAFA2 HPO prediction benchmark

Performance results (average term centric AUROC curve) of the top performing groups (grey bars), baseline classifiers (red and blue bars) and HPO2GO (dark grey bar) in CAFA2 HPO prediction benchmark.

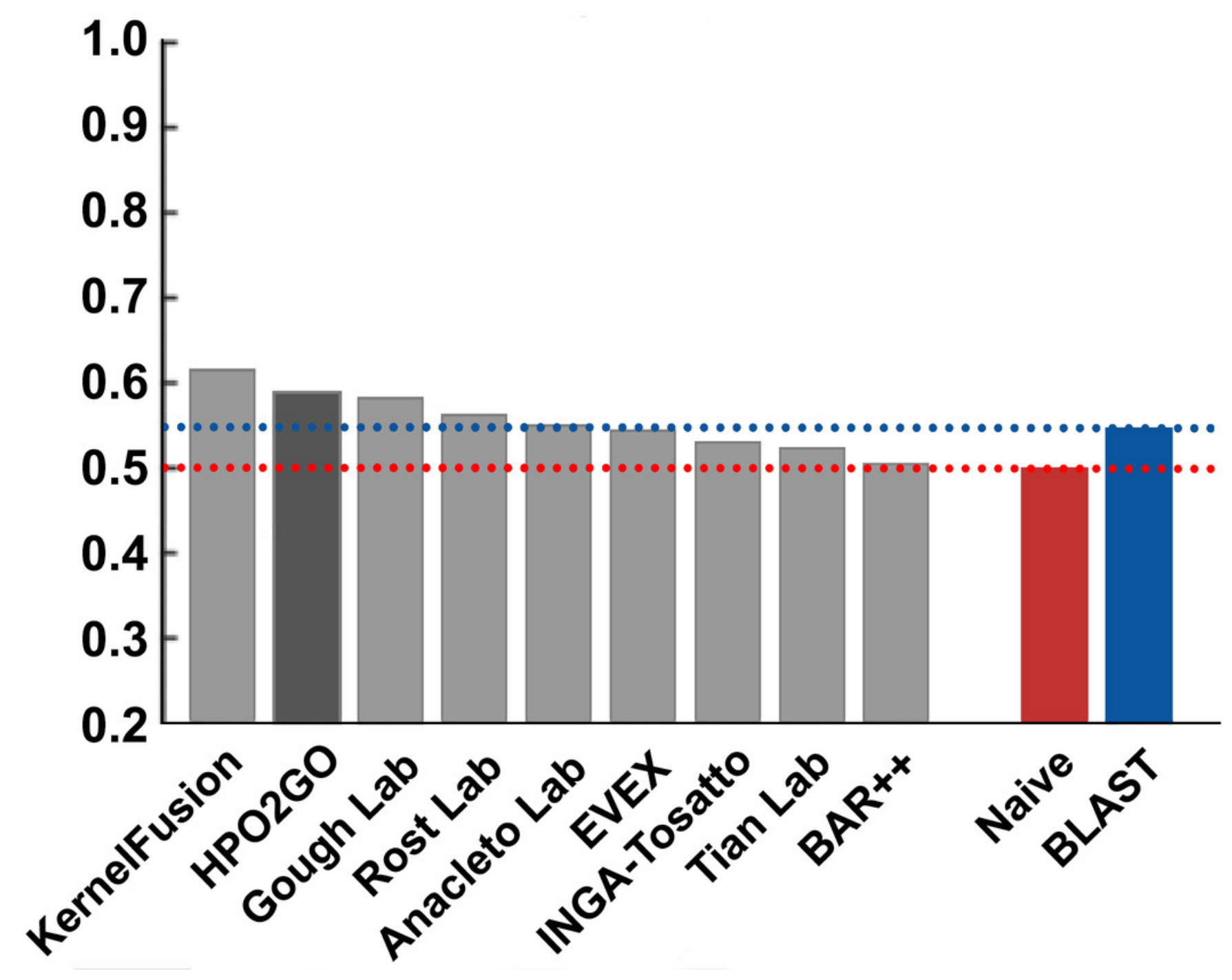

